\title{
Stability and control of radial deployment of electric solar wind sail
}

\author{
Gangqiang Li $\cdot$ Zheng H. Zhu (D) Chonggang Du
}

Received: 27 June 2020/Accepted: 30 October 2020/Published online: 5 January 2021

(C) Springer Nature B.V. 2021

\begin{abstract}
The paper studies the stability and control of radial deployment of an electric solar wind sail with the consideration of high-order modes of elastic tethers. The electric solar wind sail is modeled by combining the flexible tether dynamics, the rigid-body dynamics of central spacecraft, and the flexible-rigid kinematic coupling. The tether deployment process is modeled by the nodal position finite element method in the arbitrary Lagrangian-Eulerian framework. A symplectic-type implicit Runge-Kutta integration is proposed to solve the resulting differential-algebraic equation. A proportional-derivative control strategy is applied to stabilize the central spacecraft's attitudes to ensure tethers' stable deployment with a constant spinning rate. The results show the electric solar wind sail requires thrust at remote units in the tangential direction to counterbalance the Coriolis forces acting on the tethers and remote units to deploy tethers radially successfully. The parametric analysis shows the tether deployment speed and the thrust magnitude significantly impacts deployment stability and tether libration, which opens the possibility of successful
\end{abstract}

G. Li · Z. H. Zhu ( $ه)$

Department of Mechanical Engineering, York University, 4700 Keele Street, Toronto, ON M3J 1P3, Canada e-mail: gzhu@yorku.ca

C. Du

Department of Earth and Space Science and Engineering, York University, 4700 Keele Street, Toronto,

ON M3J 1P3, Canada deployment of tethers by using optimal control. Finally, the analysis results show that radial deployment is advantageous due to the isolated deployment mechanism, and a jammed tether can be isolated from affecting the deployment of rest tethers.

Keywords Space tether - Electric solar wind sail . Multibody dynamics · Rigid-flexible coupling · Flexible structural stability · Nodal position finite element method · Arbitrary Lagrangian-Eulerian

\section{Introduction}

Electric solar wind sail (E-sail) is an innovative propellantless propulsion technology for interplanetary exploration [1-4]. Typically, an E-sail consists of a central spacecraft connected with multiple long and thin conductive tethers in a hub-spoke like configuration [5]. The geometrical configuration is maintained and stabilized by pulling tethers radially with the centrifugal forces resulting from spinning tethers around the central spacecraft. These spinning tethers are positively charged by the central spacecraft to form an electrostatic field over a large circular area-the spin plane, which deflects the trajectory of incident protons in the solar wind to generate thrust [5-7]. Although the E-sail has been studied extensively $[2,4,8-10]$, less attention has been paid to the 
dynamic process of tether deployment from the central spacecraft. There are two deployment schemes proposed in Ref. [11] to deploy the tethers either tangentially or radially. Each deployment scheme has its pros and cons. For the tangential deployment of the flexible tethers, the tethers are prewound up on the exterior of the central spacecraft and then deployed by spinning the central spacecraft in a predefined spin trajectory. The deployment dynamics has been well studied in Refs. [12, 13]. It is noted that the jamming of any one of the tethers will cause the tether tangling leading to the failure of the deployment. In case of the radial deployment, each tether is stored in an individual spool. The tethers could be either pulled out by the thruster at the remote units at the end of tethers with an initial push by the spring forces [14] or be pushed out by individually controlled active spool and then pulled the tethers straight by spinning the central spacecraft [11]. Substantial engineering analysis has recently shown that the radial deployment scheme has the advantage of low failure risk of tether tangling over the tangential deployment scheme. However, the Coriolis force, induced by either the orbital motion of E-sail or the spin of E-sail in the latter case of radial deployment, may cause the tether librate about its equilibrium positions and eventually tangle each other. Many control laws for the space tether deployment have been proposed to suppress the libration motion caused by the Coriolis force that is induced by the deployment velocity [15-17]. Furthermore, the thruster in the remote unit at the tip of tether is proposed to deploy the tethers to their equilibrium positions faster [18]. It is noted that the central spacecraft is assumed as a lumped mass without the consideration of attitude dynamics. However, for an E-sail system, studies suggest that the attitude motion of central spacecraft should be regulated to make it rotating around its principal axis in a predefined trajectory $[6,11]$. In the current work, we assume the tethers are deployed by reeling them out of the central spacecraft. The deployed tethers are kept straight in the radial direction by spinning the central spacecraft. The reel and spin rates controlled individually to ensure the tethers are deployed in the radial direction safely.

The novelty of current work is the dynamic modeling and characterization of the radial tether deployment process considering high-order modes of elastic tethers. The central spacecraft is modeled as a six-degree-of- freedom (DOF) rigid body, while the tethers are modeled with 2-noded bar elements with variable lengths and zero compressive stiffness. Recently, three different beam models of the tether for the E-sail have been tested based on the Abaqus software, and its impact on the transient response of the tether is investigated [19]. In the current paper, all tethers are treated as flexible elastic tensile members in the deployment process. Two approaches deal with the variation of tether length in discretized tether models $[20,21]$. In the first approach, the total numbers of elements in the model are kept constant to ease programming and implementation. The tether deployment is represented by increasing the lengths of either all elements at the same rate [22] or selected elements at different rates [23]. In the second approach, the total numbers of elements in the model vary as the tethers are deployed. As the tether length increases in the deployment process, the element connected to the central spacecraft increases. Once the length of this variablelength element is longer than a preset element length, this element is divided into two elements: a variablelength element and a new constant-length element [24-26]. This process is achieved in the Arbitrary Lagrangian-Eulerian (ALE) framework [24, 25, 27]. The second approach is superior to the first approach for the current study due to the easy implementation of kinematic constraints of the connection relationship between the central spacecraft and multiple tethers. Recently, the authors proposed a nodal position finite element method in the ALE description (NPFEM_ALE) to study the variable-length tether problem in the space elevator with moving climbers [21, 28, 29]. Furthermore, the finite element model of variablelength tether embedded with the second approach is also used for the space tether systems [24, 27]. However, it is found that the previous models cannot be directly applied in the current study due to the following limitations: the neglection of orbital motion and gravity gradient along the tether [24], the neglection of the transverse flexural motion of tether [27], and the use of an inappropriate time integration scheme leading to the violation of constraint conditions at the interface of central spacecraft and tether [25, 27]. For the E-sail, the orbital motion of the central spacecraft around the Sun is at the order of $10^{-7} \mathrm{rad} / \mathrm{s}$ when the E-sail is at 1 Astronomical Unit (AU) from the Sun, which is at the same numerical 
order of the convergence tolerance of implicit solvers employed in the program, such as the first-order Backward Euler and the second-order generalized alpha methods. This leads to the violation of constraint equations in the differential equations and generates unreliable results [27]. To address these mentioned limitations, the NPFEM_ALE model of the space elevator $[21,25,28,29]$ is extended and applied to the tether deployment problem of E-sail. Furthermore, a framework of an implicit Runge-Kutta implicit time integration with $s$ stages and a symplectic property for solving the differential-algebraic equations is developed.

\section{Mathematic formulation of electric solar wind sail}

\subsection{Finite element discretization of flexible tethers}

Consider the central spacecraft shown in Fig. 1 that deploy flexible tethers by rotating about its principal axis at a constant spinning rate. The central spacecraft is modeled as a 6-DOF rigid body with attitude dynamics, while the remote units located at the tip of each tether are assumed as lumped masses without attitude dynamics. The motion of the E-sail is described in two coordinate systems, the heliocentric-ecliptic inertial frame $\boldsymbol{O}_{g} \boldsymbol{X}_{g} \boldsymbol{Y}_{g} \boldsymbol{Z}_{g}$ and the bodyfixed frame $\boldsymbol{O}_{b} \boldsymbol{X}_{b} \boldsymbol{Y}_{b} \boldsymbol{Z}_{b}$. The definition of the heliocentric-ecliptic inertial frame can be found in our previous works $[6,30]$. The origin of the body-fixed frame $\left(\boldsymbol{O}_{b} \boldsymbol{X}_{b} \boldsymbol{Y}_{b} \boldsymbol{Z}_{b}\right)$ is located at the center of mass of the central spacecraft with the $\boldsymbol{O}_{b} \boldsymbol{Z}_{b}$ axis pointing to the normal direction of spinning plane of the E-sail, the $\boldsymbol{O}_{b} \boldsymbol{Y}_{b}$ axis pointing to the direction of the cross product of $\boldsymbol{O}_{b} \boldsymbol{Z}_{b}$ and $\boldsymbol{O}_{g} \boldsymbol{Z}_{g}$, and the $\boldsymbol{O}_{b} \boldsymbol{X}_{b}$ axis completing a right-handed frame. Also, two additional frames are introduced. One is to describe the libration motion of flexible tethers with the origin at each tether's anchor points on the central spacecraft. Another is used to apply the thrust conveniently with the origin at the remote unit. Their definition will be given in the following sections.
Fig. 1 Schematic diagram of radial deployment of E-sail tethers a coordinate definition, $\mathbf{b}$ radial deployment mechanism of an E-sail

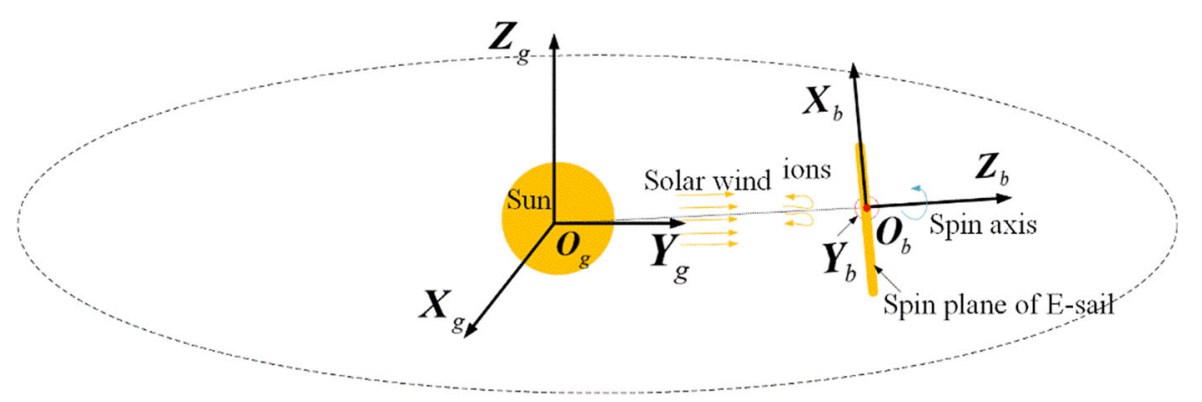

(a)

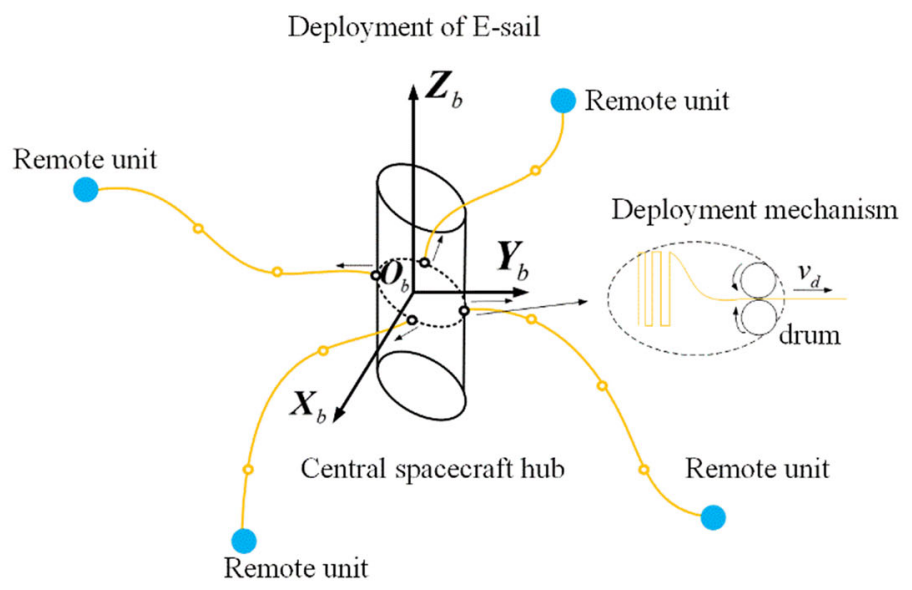

(b) 
The equations of motion (EOM) of the tethers are derived from the generalized D'Alembert principle $[20,29]$. From the virtual work principle, the sum of virtual work of all the applied and inertial forces on an arbitrary virtual displacement of the tethers should be zero at an arbitrary moment. The virtual work of the $k$ th element is written as,

$\delta W_{k}=\delta W_{e, k}+\delta W_{g, k}+\delta W_{i, k}=0$

where $\delta$ is the variational operator, and $\delta W_{e, k}, \delta W_{g, k}$, and $\delta W_{i, k}$ denote the virtual work done by the elastic, gravitational, and inertial forces, respectively.

$$
\begin{aligned}
\delta W_{e, k} & =-\int \delta \boldsymbol{\varepsilon}^{\mathrm{T}} \boldsymbol{\sigma} A_{k} \mathrm{~d} p=-\int_{p_{k}}^{p_{k+1}} A_{k} \delta \boldsymbol{\varepsilon}^{\mathrm{T}} \boldsymbol{\sigma} \mathrm{d} p \\
& =-\delta \boldsymbol{X}_{e, k}^{\mathrm{T}} \boldsymbol{F}_{e, k}
\end{aligned}
$$

$$
\begin{aligned}
\delta W_{g, k} & =\int \delta \boldsymbol{X}_{e, k}^{\mathrm{T}} \boldsymbol{f}_{g, k} A_{k} \mathrm{~d} p=\int_{p_{k}}^{p_{k+1}} \delta \boldsymbol{X}_{e, k}^{\mathrm{T}} \boldsymbol{f}_{g, k} A_{k} \mathrm{~d} p \\
& =\delta \boldsymbol{X}_{e, k}^{\mathrm{T}} \boldsymbol{F}_{g, k}
\end{aligned}
$$

$$
\begin{aligned}
\delta W_{i, k}= & \int \delta \boldsymbol{X}_{e, k}^{\mathrm{T}}\left(-\rho_{k} A_{k} \ddot{\boldsymbol{X}}\right) \mathrm{d} p= \\
& -\int_{p_{k}}^{p_{k+1}} \delta \boldsymbol{X}_{e, k}^{\mathrm{T}} \rho_{k} A_{k} \ddot{\boldsymbol{X}} \mathrm{d} p \\
= & -\delta \boldsymbol{X}_{e, k}^{\mathrm{T}}\left(\boldsymbol{M}_{e, k} \ddot{\boldsymbol{X}}_{e, k}+\boldsymbol{F}_{p, k}\right)
\end{aligned}
$$

$$
\ddot{\boldsymbol{X}}=\boldsymbol{N}_{e, k} \ddot{\boldsymbol{X}}_{e, k}+\boldsymbol{a}_{p, k}
$$$$
\begin{aligned}
\boldsymbol{N}_{e, k}= & \left(\frac{1-\xi(t)}{2} \boldsymbol{I}, \frac{\partial \boldsymbol{N}_{a, k}}{\partial p_{k}} \boldsymbol{X}_{a, k}, \frac{1+\xi(t)}{2} \boldsymbol{I}, \frac{\partial \boldsymbol{N}_{a, k}}{\partial p_{k+1}} \boldsymbol{X}_{a, k}\right) \\
& \text { and } \quad \boldsymbol{N}_{a, k}=\left[\frac{1-\xi(t)}{2} \boldsymbol{I}, \frac{1+\xi(t)}{2} \boldsymbol{I}\right]
\end{aligned}
$$

$$
\begin{aligned}
\boldsymbol{a}_{p, k}= & 2\left(\frac{\partial \boldsymbol{N}_{a, k}}{\partial p_{k}} \dot{p}_{k}+\frac{\partial \boldsymbol{N}_{a, k}}{\partial p_{k+1}} \dot{p}_{k+1}\right) \dot{\boldsymbol{X}}_{a, k} \\
& +\left(\frac{\partial^{2} \boldsymbol{N}_{a, k}}{\partial p_{k}^{2}} \dot{p}_{k}^{2}+2 \frac{\partial^{2} \boldsymbol{N}_{a, k}}{\partial p_{k} \partial p_{k+1}} \dot{p}_{k} \dot{p}_{k+1}+\frac{\partial^{2} \boldsymbol{N}_{a, k}}{\partial p_{k+1}^{2}} \dot{p}_{k+1}^{2}\right) \boldsymbol{X}_{a, k}
\end{aligned}
$$

where $\quad \boldsymbol{X}_{a, k}=\left(X_{k}, Y_{k}, Z_{k}, X_{k+1}, Y_{k+1}, Z_{k+1}\right)^{\mathrm{T}} \quad$ and $\boldsymbol{X}_{e, k}=\left(X_{k}, Y_{k}, Z_{k}, p_{k}, X_{k+1}, Y_{k+1}, Z_{k+1}, p_{k+1}\right)^{\mathrm{T}}$ are the position vectors of element nodes and material point, respectively, $p_{j}(j=k, k+1)$ is the material coordinate with the subscript indicating the connecting node, $\xi(t)=s / L_{e, k}(-1 \leq \xi(t) \leq 1)$ with $s$ being the arc- length of the $k$ th element measured from the beginning of each element, $\boldsymbol{I}$ is a diagonal identity matrix with $3 \times 3$ dimension, $L_{e}$ is the stretched length, $\rho$ and $A$ are the material density and cross-section area of element, $\boldsymbol{\sigma}$ and $\boldsymbol{\varepsilon}$ are the vectors of stress and strain, respectively, $\boldsymbol{f}_{g, k}$ is the vector of gravitational force per unit length. The overhead dots $(\dot{)}$ and $\ddot{()}$ denote the firstand second-order time derivatives.

Substituting Eqs. (2)-(7) into Eq. (1) yields,

$\boldsymbol{M}_{e, k} \ddot{\boldsymbol{X}}_{e, k}=\boldsymbol{F}_{e, k}+\boldsymbol{F}_{g, k}-\boldsymbol{F}_{p, k}$

with

$$
\left\{\begin{array}{l}
\boldsymbol{M}_{e, k}=\frac{L_{p, k}}{2} \int_{-1}^{1} \rho_{k} A_{k} \boldsymbol{N}_{e, k}^{\mathrm{T}} \boldsymbol{N}_{e, k} \mathrm{~d} \xi \\
\boldsymbol{F}_{e, k}=\frac{L_{p, k}}{2} \int_{-1}^{1} E_{k} A_{k} \varepsilon\left(\frac{\partial \varepsilon}{\partial \boldsymbol{X}_{e, k}}\right)^{\mathrm{T}} \mathrm{d} \xi \\
\boldsymbol{F}_{g, k}=\frac{L_{p, k}}{2} \int_{-1}^{1} \boldsymbol{N}_{e, k}^{\mathrm{T}} \boldsymbol{f}_{g, k} \mathrm{~d} \xi \\
\boldsymbol{F}_{p, k}=\frac{L_{p, k}}{2} \int_{-1}^{1} \rho_{k} A_{k} \boldsymbol{N}_{e, k}^{\mathrm{T}} \boldsymbol{a}_{p, k} \mathrm{~d} \xi
\end{array}\right.
$$

where $\boldsymbol{M}_{\mathrm{e}}$ is the mass matrix of element, $\boldsymbol{F}_{\mathrm{e}}$ and $\boldsymbol{F}_{\mathrm{g}}$ are the force vectors of the elastic and gravitational forces, respectively, $\boldsymbol{F}_{\mathrm{p}, \mathrm{t}}$ is the force vector caused by the mass transportation across the element boundaries due to tether deployment, which vanishes when the material coordinate of the tether is fixed $[21,28,29,31] . L_{p, k}=p_{k+1}-p_{k}$ is the element length due to variation of material coordinate. In the current paper, the tether damping is not considered due to the lack of data in space [25].

For an E-sail containing $m \geq 2$ tethers as shown in Fig. 1, the tether is divided into $n$ elements with $n+1$ nodes. Thus, there are a total of $m \times n$ tether elements. By assembling the EOM of all elements with the standard procedure in the finite element method $[32,33]$, the EOMs of all tethers become,

$$
\boldsymbol{M}_{\mathrm{t}} \ddot{\boldsymbol{X}}_{\mathrm{t}}=\boldsymbol{F}_{\mathrm{e}}+\boldsymbol{F}_{\mathrm{g}}-\boldsymbol{F}_{\mathrm{p}}
$$

where $\boldsymbol{M}_{\mathrm{t}}$ is the mass matrix of tethers with detailed form in Ref. [25], $\boldsymbol{X}_{\mathrm{t}}$ is the position vector of tethers, $\boldsymbol{F}_{\mathrm{e}}, \boldsymbol{F}_{\mathrm{g}}$, and $\boldsymbol{F}_{\mathrm{p}}$ are the force vectors acting on tethers $[21,28]$. The lumped masses of the remote units are added into the mass matrix $\boldsymbol{M}_{\mathrm{t}}$ of the tethers in the corresponding places for the connecting nodes [25]. 
2.2 Translation and attitude motions of the central spacecraft

The central spacecraft is assumed as a 6 DOF rigid body. First, the EOM of translation dynamics of the central spacecraft is derived in the global inertial coordinate system by Newton's second law directly,

$\boldsymbol{M}_{s} \ddot{\boldsymbol{X}}_{s}=\boldsymbol{F}_{s, g}+\boldsymbol{F}_{s, p}$

where $\boldsymbol{M}_{s}=\operatorname{diag}\left(m_{s}, m_{s}, m_{s}\right)$ is the mass matrix of the central spacecraft with $m_{s}$ being the mass of the central spacecraft, $\boldsymbol{X}_{s}=\left(X_{s}, Y_{s}, Z_{s}\right)^{\mathrm{T}}$ is the position vector of the center of mass of the central spacecraft with overhead dot representing time derivative, while $\boldsymbol{F}_{s, g}$ and $\boldsymbol{F}_{s, p}$ are the external force vectors due to the gravitational and other perturbative forces, respectively.

The equation of attitude motion of the central spacecraft is described in the body-fixed coordinate system $\boldsymbol{O}_{b} \boldsymbol{X}_{b} \boldsymbol{Y}_{b} \boldsymbol{Z}_{b}$ via the Euler's equations of motion [27],

$$
\begin{aligned}
& \dot{\boldsymbol{\theta}}=\boldsymbol{J}(\boldsymbol{\theta}) \boldsymbol{\omega} \\
& \boldsymbol{H}_{s} \dot{\boldsymbol{\omega}}+\boldsymbol{\omega} \times\left(\boldsymbol{H}_{s} \boldsymbol{\omega}\right)=\boldsymbol{M}_{s, g}+\boldsymbol{M}_{s, p}+\boldsymbol{M}_{s, c} \\
& \boldsymbol{J}(\boldsymbol{\theta})=\frac{1}{\mathrm{c} \theta}\left(\begin{array}{ccc}
\mathrm{c} \theta & \mathrm{s} \phi \mathrm{s} \theta & \mathrm{c} \phi \mathrm{s} \theta \\
0 & \mathrm{c} \phi \mathrm{c} \theta & -\mathrm{s} \phi \mathrm{c} \theta \\
0 & \mathrm{~s} \phi & \mathrm{c} \phi
\end{array}\right)
\end{aligned}
$$

where $\boldsymbol{\theta}=(\phi, \theta, \psi)^{\mathrm{T}}$ is the Euler angle vector with $\phi$, $\theta$, and $\psi$ denoting the roll, pitch, and yaw angle, respectively. It is noted that the singularity condition, $\theta=90^{\circ}$, will never happens. $\omega=\left(\omega_{x}, \omega_{y}, \omega_{z}\right)^{\mathrm{T}}$ is the angular velocity vector, $\boldsymbol{H}_{s}=\operatorname{diag}\left(I_{X}, I_{Y}, I_{Z}\right)$ is the angular inertial matrix of the central spacecraft with $I_{X_{b}}=I_{Y_{b}}=\frac{1}{12} m_{s}\left(3 r_{s}^{2}+h^{2}\right)$ and $I_{Z_{b}}=\frac{1}{2} m_{s} r_{s}^{2}, r_{s}$ and $h$ are the radius and height of the cylindrical central spacecraft, respectively. $\boldsymbol{M}_{s, g}, \boldsymbol{M}_{s, p}$ and $\boldsymbol{M}_{s, t}$ are the torque vectors due to the gravitational, perturbative, and control input forces, respectively. The symbols $c$ and $s$ in Eq. (13) represent the cosine and sine functions.

\subsection{Coupling constraint equations}

between the tether and central spacecraft

The constraint equations due to the kinematic coupling between the tethers and the central spacecraft are written as,

$$
\boldsymbol{C}_{1}=\boldsymbol{X}_{s}+\boldsymbol{T}_{b 2 g}(\boldsymbol{\theta}) \boldsymbol{X}_{\mathrm{anc}, j}^{b}-\boldsymbol{X}_{t, j}=\boldsymbol{O}(j=1, \ldots, m)
$$

$$
\boldsymbol{T}_{b 2 g}(\boldsymbol{\theta})=\left[\begin{array}{ccc}
c \psi c \theta & c \psi s \theta s \phi-c \phi s \psi & c \phi c \psi+s \phi s \psi \\
c \theta s \psi & c \phi c \psi+s \theta s \phi s \psi & -c \psi s \phi+c \phi s \theta s \psi \\
-s \theta & c \theta s \phi & c \theta c \phi
\end{array}\right]
$$

where $\boldsymbol{X}_{\mathrm{anc}, j}^{b}$ is the position vector of anchor points with the superscript $b$ representing the body-fixed frame and the subscript $j=(1 \sim m)$ representing the number index of the tether. They will be given in numerical simulation section. $\boldsymbol{T}_{b 2 g}(\boldsymbol{\theta})$ is the transformation matrix of the central spacecraft from the bodyfixed frame $O_{b} \boldsymbol{X}_{b} \boldsymbol{Y}_{b} \boldsymbol{Z}_{b}$ to the global inertial frame $O_{g} \boldsymbol{X}_{g} \boldsymbol{Y}_{g} \boldsymbol{Z}_{g}, \boldsymbol{X}_{t, j}$ is the position vector of anchor point for the $j$ th tether in the global inertial frame.

\subsection{Constraint equations for tethers}

There are two types of tether nodes used in the proposed model: the moving node and the normal finite element node. The moving node represents the tether deployment process [21, 29]. As shown in Fig. 1, a drum-type deployment mechanism is assumed here, where two drums are used to control a tether's deploying speed. It is assumed (1) there is no slippage between the drum and the tether, (2) the momentum of the rotating drum does not affect the attitudes of the central spacecraft, and (3) all tethers are deployed at the same speed. The tether deployment process is modeled as the following: (1) the nodes of tethers connecting to the central spacecraft are defined as the moving nodes, (2) the material coordinate $p$ of the moving node follows a prescribed trajectory or deploying speed. The constraint equations for the moving nodes are defined as,

$\boldsymbol{C}_{2}=p_{j}-p_{j, \mathrm{pre}}=\boldsymbol{0}$

where $p_{j, \text { pre }}=p_{j, \text { ini }}+\dot{p}_{j, \text { pre }} \Delta t$ is the material coordinate of a predefined trajectory with the subscript $j$ representing the $j$ th node. Here, for simplicity, the tether is deployed at a constant speed $\dot{p}_{j \text {,pre }}$, where $\dot{p}_{j, \text { pre }}<0$ represents the tether deployment, and $\dot{p}_{j, \text { pre }}>0$ represents the tether retrieval.

For the self-content and remodeling purpose for the interested readers, the necessary process of dividing an element is given here. The interested reader can find 
detailed information from our previous works [21, 25, 29]. As shown in Fig. 2a, where the E-sail is connected with four tethers, the nodes of anchor points of the tethers are defined as the moving nodes, and the elements connected to the moving nodes at one end are defined as the variable-length elements. The lengths of variable-length elements increase at the spacecraft side as the tethers are deployed out from the central spacecraft. Once the variable-length element's length exceeds a preset value, the element will be divided into a variable-length element and a constant-length element by adding one node.

In the deployment process, only the dividing of elements happens. Two parameters, the standard $L_{s}$ and upper bound $\left(L_{\max }\right)$ lengths are defined for this process. The variable-length element will be divided into two elements if its length exceeds the upper bound $L_{\text {max }}$. For example, as shown in Fig. $2 b$, the first node is a moving node representing the tether deployed out from the central spacecraft in the arrow direction. If the condition

$L_{1}=p_{2}-p_{1} \geq L_{\max }$

is satisfied, then the variable-length element is divided into two new elements by adding a new node between the first and second nodes. The position, velocity, and acceleration of the newly added node are obtained by linear interpolation. After that, the nodes and elements after the second node must be renumbered. The length of the new variable-length element is $L_{1}-L_{s}$, and the second new element is a constant-length element with a standard length $L_{s}$.

Moreover, the mass conservation at the central spacecraft should satisfy the following equation to account for the loss or gain of mass by the deployment of tether, such that,

$m_{s}=m_{s, \text { init }}+\sum_{j=1}^{m} \dot{p}_{j, \text { pre }} A_{j} \rho_{j} \Delta t$

where $m_{s, \text { init }}$ is the mass of the central spacecraft before the deployment, $m$ represents the number of tethers while $A$ and $\rho$ are the cross-section area and material density of tethers.

The process of dividing of element happens simultaneously for each tether when the deployed speed of each tether is the same. So the standard lengths of each tether $L_{s_{j}}(j=1, \ldots, m)$ are set slightly different $L_{s_{j}}=$ $[1+0.01(j-1)] L_{S_{1}}(j=2, \ldots, m)$ to avoid the phenomenon.

Except for the moving nodes, all other nodes of tethers are the normal nodes in the finite element method with constant material coordinates [24, 25]. The constraint equations of these normal nodes are,

$\boldsymbol{C}_{3}=p_{j}-p_{j, \text { ini }}=\mathbf{0}$

where $p_{j \text {,ini }}$ is the material coordinate of $j$ th normal node with the subscript "ini" indicating the constant.

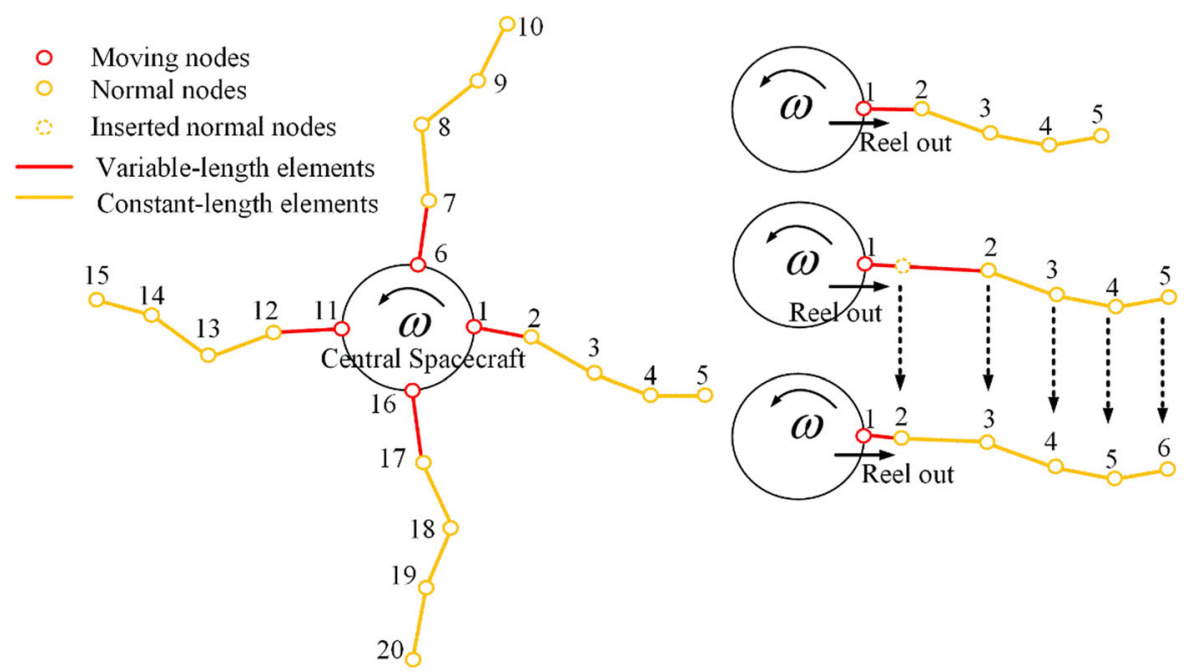

(a)

(b)

Fig. 2 Illustration of dividing of tether elements 


\subsection{Equation of motion of the E-sail}

The EOM of the E-sail is obtained by combining Eqs. (8)-(19) by the Lagrangian multiplier method, that is,

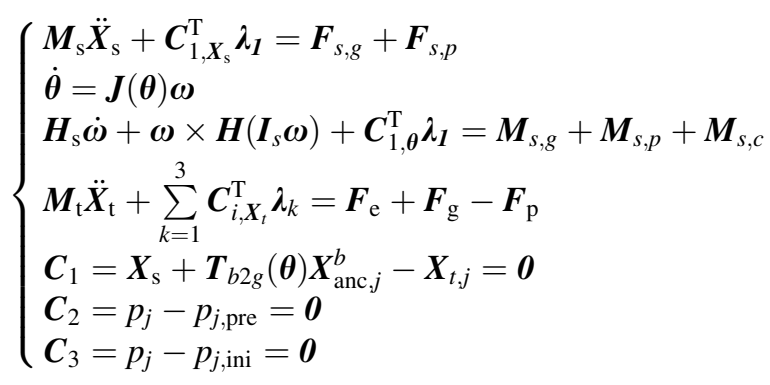

where $\boldsymbol{C}_{1, \boldsymbol{X}_{\mathrm{s}}}^{\mathrm{T}}=\left(\partial \boldsymbol{C}_{1} / \partial \boldsymbol{X}_{\mathrm{s}}\right)^{\mathrm{T}}$ is the Jacobian matrix of constraint equation with respect to the position vector of the central spacecraft, the superscript $T$ represents the transpose of a matrix, $\boldsymbol{C}_{1, \theta}^{\mathrm{T}}$ is the Jacobian matrix of the constraint equation with respect to the attitude of the central spacecraft, $\quad \boldsymbol{C}_{k, \boldsymbol{X}_{\mathrm{s}}}^{\mathrm{T}}=$ $\left(\partial C_{k} / \partial \boldsymbol{X}_{t}\right)^{\mathrm{T}}(k=1,2,3)$ is the Jacobian matrix of constraint equations with respect to the position vectors of the tether, and $\lambda_{k}(k=1,2,3)$ are the Lagrangian multiplier vectors.

\section{Time integration method}

The differential-algebraic equations in Eq. (20) are solved numerically by an implicit $s$-stage RungeKutta integration scheme with symplectic property, which ensures the numerical model's stability or energy conservation for the long-term integration process [20]. However, Ref. [20] applied the implicit $s$-stage Runge-Kutta integration scheme to solve the differential equations of tethers without constraint equations and requires the inverse of the tether's mass matrix. With the introduction of material coordinate $p$, the mass matrix of tethers is rank-deficient and not invertible. Thus, a Newton-Raphson iteration algorithm is used to solve these algebraic equations [32]. The scheme is explained as follows.

First, the second-order differential equations in Eq. (20) is reduced to the first-order differential equations, that is,

$$
\left\{\begin{array}{l}
\dot{\boldsymbol{X}}_{\mathrm{s}}=\boldsymbol{V}_{\mathrm{s}} \\
\boldsymbol{M}_{\mathrm{s}} \dot{\boldsymbol{V}}_{\mathrm{s}}+\boldsymbol{C}_{1, \boldsymbol{X}_{\mathrm{s}}}^{\mathrm{T}} \boldsymbol{\lambda}_{\boldsymbol{I}}=\boldsymbol{F}_{s, g}+\boldsymbol{F}_{s, p} \\
\dot{\boldsymbol{\theta}}=\boldsymbol{J}(\boldsymbol{\theta}) \boldsymbol{\omega} \\
\boldsymbol{H}_{\mathrm{s}} \dot{\boldsymbol{\omega}}+\boldsymbol{\omega} \times\left(\boldsymbol{H}_{\mathrm{s}} \boldsymbol{\omega}\right)+\boldsymbol{C}_{1, \boldsymbol{\theta}}^{\mathrm{T}} \boldsymbol{\lambda}_{\boldsymbol{I}}=\boldsymbol{M}_{s, g}+\boldsymbol{M}_{s, p}+\boldsymbol{M}_{s, c} \\
\dot{\boldsymbol{X}}_{t}=\boldsymbol{V}_{t} \\
\boldsymbol{M}_{\mathrm{t}} \dot{\boldsymbol{V}}_{\mathrm{t}}+\sum_{l=1}^{3} \boldsymbol{C}_{l, \boldsymbol{X}_{\mathrm{t}}}^{\mathrm{T}} \boldsymbol{\lambda}_{l}=\boldsymbol{F}_{\mathrm{e}}+\boldsymbol{F}_{\mathrm{g}}-\boldsymbol{F}_{\mathrm{p}} \\
\boldsymbol{C}_{1}=\boldsymbol{X}_{\mathrm{s}}+\boldsymbol{T}_{b 2 g}(\boldsymbol{\theta}) \boldsymbol{X}_{\mathrm{anc}, j}^{b}-\boldsymbol{X}_{t, j}=\boldsymbol{O} \\
\boldsymbol{C}_{2}=p_{j}-p_{j, \mathrm{pre}}=\boldsymbol{0} \\
\boldsymbol{C}_{3}=p_{j}-p_{j, \mathrm{ini}}=\mathbf{0}
\end{array}\right.
$$

where $\boldsymbol{V}_{\mathrm{s}}$ and $\boldsymbol{V}_{\mathrm{t}}$ represent the velocity vectors of the central spacecraft and tether, respectively.

Second, define a new vector $\boldsymbol{Z}=$ $\left(\boldsymbol{X}_{\mathrm{s}}, \boldsymbol{V}_{\mathrm{s}}, \boldsymbol{\theta}, \boldsymbol{\omega}, \boldsymbol{X}_{\mathrm{t}}, \boldsymbol{V}_{\mathrm{t}}\right)^{\mathrm{T}}$ to simplify Eq. (21) as,

$\left\{\begin{array}{l}\boldsymbol{A}(\boldsymbol{Z}) \boldsymbol{Z}=\boldsymbol{f}(\boldsymbol{Z}) \\ \boldsymbol{C}(\boldsymbol{Z})=\boldsymbol{0}\end{array}\right.$

where

$$
\begin{aligned}
\boldsymbol{A} & =\left(\begin{array}{cccccc}
\boldsymbol{I} & \boldsymbol{0} & \boldsymbol{0} & \boldsymbol{0} & \boldsymbol{0} & \boldsymbol{0} \\
\boldsymbol{0} & \boldsymbol{M}_{\mathrm{s}} & \boldsymbol{0} & \boldsymbol{0} & \boldsymbol{0} & \boldsymbol{0} \\
\boldsymbol{0} & \boldsymbol{0} & \boldsymbol{I} & \boldsymbol{0} & \boldsymbol{0} & \boldsymbol{0} \\
\boldsymbol{0} & \boldsymbol{0} & \boldsymbol{0} & \boldsymbol{H}_{\mathrm{s}} & \boldsymbol{0} & \boldsymbol{0} \\
\boldsymbol{0} & \boldsymbol{0} & \boldsymbol{0} & \boldsymbol{0} & \boldsymbol{I} & \boldsymbol{0} \\
\boldsymbol{0} & \boldsymbol{0} & \boldsymbol{0} & \boldsymbol{0} & \boldsymbol{0} & \boldsymbol{M}_{\mathrm{t}}
\end{array}\right) \text { and } \\
& \left\{\begin{array}{l}
\boldsymbol{f}_{1}(\boldsymbol{Z})=\boldsymbol{V}_{\mathrm{s}} \\
\boldsymbol{f}_{2}(\boldsymbol{Z})=\boldsymbol{F}_{s, g}+\boldsymbol{F}_{s, p}-\boldsymbol{C}_{1, \boldsymbol{X}_{s}}^{\mathrm{T}} \boldsymbol{\lambda}_{\boldsymbol{I}} \\
\boldsymbol{f}_{3}(\boldsymbol{Z})=\boldsymbol{J}(\boldsymbol{\theta}) \boldsymbol{\omega} \\
\boldsymbol{f}_{4}(\boldsymbol{Z})=\boldsymbol{M}_{s, g}+\boldsymbol{M}_{s, c}+\boldsymbol{M}_{s, p}-\boldsymbol{\omega} \times\left(\boldsymbol{H}_{\mathrm{s}} \boldsymbol{\omega}\right)-\boldsymbol{C}_{1, \theta}^{\mathrm{T}} \boldsymbol{\lambda}_{\boldsymbol{I}} \\
\boldsymbol{f}_{5}(\boldsymbol{Z})=\boldsymbol{V}_{\mathrm{t}} \\
\boldsymbol{f}_{6}(\boldsymbol{Z})=\boldsymbol{F}_{\mathrm{e}}+\boldsymbol{F}_{\mathrm{g}}-\boldsymbol{F}_{\mathrm{p}}-\sum_{l=1}^{3} \boldsymbol{C}_{l, \boldsymbol{X}_{t}}^{\mathrm{T}} \boldsymbol{\lambda}_{l}
\end{array}\right.
\end{aligned}
$$

It should be pointed out that the matrix $\boldsymbol{A}$ in Eq. (23) is a singular matrix due to the rank-deficient submatrix $\boldsymbol{M}_{t}$ [28]. Thus, the matrix $\boldsymbol{A}$ is not invertible in a traditional way [20], and the implicit $s$-stage RungeKutta integrator is used, that is, 


$$
\left\{\begin{array}{l}
\boldsymbol{g}_{1}=\boldsymbol{A}\left(t^{n}+c_{1} \Delta t, \boldsymbol{Z}^{n}+\Delta t \sum_{j}^{s} a_{1, j} \boldsymbol{K}_{j}\right) \boldsymbol{K}_{1}-f\left(t^{n}+c_{s} \Delta t, \mathbf{Z}^{n}+\Delta t \sum_{j}^{s} a_{1, j} \boldsymbol{K}_{j}\right)=\boldsymbol{0} \\
\vdots \\
\boldsymbol{g}_{s}=\boldsymbol{A}\left(t^{n}+c_{s} \Delta t, \mathbf{Z}^{n}+\Delta t \sum_{j}^{s} a_{s, j} \boldsymbol{K}_{j}\right) \boldsymbol{K}_{s}-f\left(t^{n}+c_{s} \Delta t, \mathbf{Z}^{n}+\Delta t \sum_{j}^{s} a_{s, j} \boldsymbol{K}_{j}\right)=\mathbf{0} \\
\boldsymbol{g}_{s+1}=\frac{\partial \boldsymbol{C}}{\partial \boldsymbol{Z}} \boldsymbol{K}_{1}=\mathbf{0} \\
\vdots \\
\boldsymbol{g}_{s+s}=\frac{\partial \boldsymbol{C}}{\partial \boldsymbol{Z}} \boldsymbol{K}_{s}=\mathbf{0}
\end{array}\right.
$$

where $\Delta t$ is the time step size, $a_{i, j}(i, j=1, \ldots, s)$ is the integration matrix, and $c_{i}(i=1, \ldots, s)$ is the integration nodes [20]. Their values are taken from the Butcher tableau form, i.e.

\begin{tabular}{c|cccc}
$c_{1}$ & $a_{11}$ & $a_{12}$ & $\cdots$ & $a_{1 s}$ \\
$c_{1}$ & $a_{21}$ & $a_{22}$ & $\cdots$ & $a_{2 s}$ \\
$\vdots$ & $\vdots$ & $\vdots$ & $\ddots$ & $\vdots$ \\
$c_{s}$ & $a_{s 1}$ & $a_{s 2}$ & $\cdots$ & $a_{s s}$ \\
\hline \multicolumn{1}{c}{$b_{1}$} & $b_{2}$ & $\cdots$ & $b_{s}$
\end{tabular}

The algebraic equation (24) with unknown $\boldsymbol{B}=$ $\left(\boldsymbol{K}_{1}, \cdots, \boldsymbol{K}_{s}\right)$ is solved by the Newton-Raphson iterative algorithm. Denote $\boldsymbol{B}^{q}$ as an approximate solution after the $q$ th iteration, the true solution can be written as,

$\boldsymbol{B}_{\text {true }}=\boldsymbol{B}^{q}+\Delta \boldsymbol{B}^{q}$

where the $\Delta \boldsymbol{B}^{q}$ is the correction to the approximate solution.

Substituting Eq. (26) into (24) and expanding it into a Taylor series by ignoring higher-order terms yield,

$\boldsymbol{O}=\boldsymbol{g}(\boldsymbol{B})=\boldsymbol{g}\left(\boldsymbol{B}^{q}+\Delta \boldsymbol{B}^{q}\right) \approx \boldsymbol{g}\left(\boldsymbol{B}^{q}\right)+\boldsymbol{J}^{q} \Delta \boldsymbol{B}^{q}$

where $\boldsymbol{J}^{q}=\partial \boldsymbol{g} /\left.\partial \boldsymbol{B}\right|_{\boldsymbol{B}^{q}}$ is the Jacobian matrix of these algebraic equations with respect to the vector $\boldsymbol{B}^{q}$. The detailed expressions of the Jacobian matrix $\boldsymbol{J}^{q}$ are given in "Appendix A". Solve for the correction $\Delta \boldsymbol{B}^{q}$ iteratively until the residual converges $\varepsilon=$ $\left|\boldsymbol{g}\left(\boldsymbol{B}_{2(4 q(n+1)+24)}^{q+1}\right)\right| /(2(4 q(n+1)+24)) \leq \varepsilon_{\max } \quad$ or the iteration number reaches the maximum allowed iteration $q_{\max }$.

Once the solution $\boldsymbol{B}=\left(\boldsymbol{K}_{1}, \ldots, \boldsymbol{K}_{s}\right)$ is obtained, the state in the next time step $\boldsymbol{Z}^{n+1}$ becomes,

$\boldsymbol{Z}^{n+1}=\boldsymbol{Z}^{n}+\Delta t \sum_{j}^{s} b_{j} \boldsymbol{K}_{j}$ where $b_{i}(i=1, \cdots, s)$ are the weight coefficients, and they are taken from Eq. (25) [20].

\section{Attitude controller for the central spacecraft}

In this section, a proportional-derivative (PD) control strategy is developed to ensure the central spacecraft's spinning rate remains stable in the tether deployment process. Rewrite the first equation in Eq. (12) as,

$\omega=J_{1}(\boldsymbol{\theta}) \dot{\theta}$

where $\boldsymbol{J}_{1}(\boldsymbol{\theta})=\boldsymbol{J}^{-1}(\boldsymbol{\theta})$.

Substituting Eq. (29) into the second equation of Eq. (12) gives,

$\tilde{\boldsymbol{M}}(\boldsymbol{\theta}) \ddot{\boldsymbol{\theta}}+\tilde{\boldsymbol{N}}(\boldsymbol{\theta}) \dot{\boldsymbol{\theta}}=\boldsymbol{u}$

with

$$
\left\{\begin{array}{l}
\tilde{\boldsymbol{M}}(\boldsymbol{\theta})=\boldsymbol{J}_{1}^{\mathrm{T}}(\boldsymbol{\theta}) \boldsymbol{H}_{\mathrm{s}} \boldsymbol{J}_{1}(\boldsymbol{\theta}) \\
\tilde{\boldsymbol{N}}(\boldsymbol{\theta})=\boldsymbol{J}_{1}^{\mathrm{T}}(\boldsymbol{\theta}) \boldsymbol{H}_{\mathrm{s}} \dot{\boldsymbol{J}}_{1}(\boldsymbol{\theta})+\boldsymbol{J}_{1}^{\mathrm{T}}(\boldsymbol{\theta}) \boldsymbol{S}\left(\boldsymbol{J}_{1}(\boldsymbol{\theta}) \dot{\boldsymbol{\theta}}\right) \boldsymbol{H}_{\mathrm{s}} \boldsymbol{J}_{1}(\boldsymbol{\theta}) \\
\boldsymbol{u}=\boldsymbol{J}_{1}^{\mathrm{T}}(\boldsymbol{\theta})\left(\boldsymbol{M}_{s, g}+\boldsymbol{M}_{s, p}+\boldsymbol{M}_{s, c}\right)
\end{array}\right.
$$

where $\boldsymbol{S}()$ is used to represent the skew-symmetric matrix [34].

Define the attitude errors of the central spacecraft as,

$\left\{\begin{array}{l}e_{\theta}=\boldsymbol{\theta}-\boldsymbol{\theta}_{d} \\ \dot{e}_{\theta}=\ddot{\boldsymbol{\theta}}-\dot{\boldsymbol{\theta}}_{d} \\ \ddot{e}_{\theta}=\ddot{\boldsymbol{\theta}}-\ddot{\boldsymbol{\theta}}_{d}\end{array}\right.$

where the subscript $d$ denotes the desired value.

Substituting Eq. (32) into Eq. (30) yields,

$\tilde{\boldsymbol{M}}(\boldsymbol{e}) \ddot{\boldsymbol{e}}+\tilde{\boldsymbol{N}}(\boldsymbol{e}) \dot{\boldsymbol{e}}=\boldsymbol{u}^{\prime}$

where $\boldsymbol{u}^{\prime}=\boldsymbol{u}-\tilde{\boldsymbol{M}} \ddot{\theta}_{d}-\tilde{\boldsymbol{N}} \dot{\theta}_{d}$.

Define the PD controller for Eq. (33) is as for Ref. [34], that is

$\boldsymbol{u}^{\prime}=-\boldsymbol{K}_{P} \boldsymbol{e}-\boldsymbol{K}_{D} \dot{\boldsymbol{e}}$

where $\boldsymbol{K}_{P}$ and $\boldsymbol{K}_{D}$ are gain matrices of the proportional and derivative terms, respectively. The detailed expression will be given in the numerical simulation part.

Substituting Eq. (34) into Eq. (31) yields the control torque $\boldsymbol{M}_{s, c}$ of the central spacecraft, 


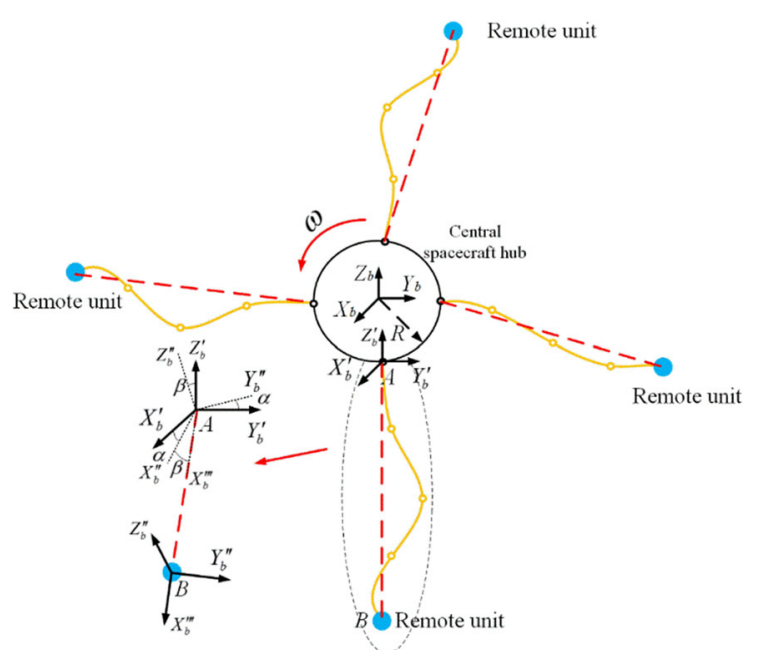

Fig. 3 Libration motion of flexible tethers of the E-sail and their simplified expressions

$$
\begin{aligned}
\boldsymbol{M}_{s, c}= & \boldsymbol{J}\left[-\boldsymbol{K}_{v}\left(\dot{\boldsymbol{\theta}}-\dot{\boldsymbol{\theta}}_{d}\right)-\boldsymbol{K}_{p}\left(\boldsymbol{\theta}-\boldsymbol{\theta}_{d}\right)+\tilde{\boldsymbol{M}} \ddot{\theta}_{d}+\tilde{\boldsymbol{N}} \dot{\theta}_{d}\right] \\
& -\boldsymbol{M}_{s, g}-\boldsymbol{M}_{s, p}
\end{aligned}
$$

Here, the desired attitude trajectory of the central spacecraft $\left(\boldsymbol{\theta}_{d}, \dot{\boldsymbol{\theta}}_{d}, \ddot{\boldsymbol{\theta}}_{d}\right)$ is calculated for the given desired angular velocity $\omega_{d}$ at the internal stages of the $s$-stage Runge-Kutta integration method,
Table 2 Position vectors of anchor points in the body-fixed frame

\begin{tabular}{ll}
\hline Name & Values \\
\hline $\boldsymbol{X}_{\text {anc }, 1}^{b}$ (m) & $(2,0,0)$ \\
$\boldsymbol{X}_{\text {anc }, 2}^{b}$ (m) & $(0,2,0)$ \\
$\boldsymbol{X}_{\text {anc }, 3}^{b}$ (m) & $(-2,0,0)$ \\
$\boldsymbol{X}_{\text {anc }, 4}^{b}(\mathrm{~m})$ & $(0,-2,0)$ \\
\hline
\end{tabular}

$\boldsymbol{\theta}_{d, j}=\boldsymbol{\theta}_{d}\left(t^{n}+c_{j} \Delta t\right), \dot{\boldsymbol{\theta}}_{d, j}=\dot{\boldsymbol{\theta}}_{d}\left(t^{n}+c_{j} \Delta t\right), \quad$ and
$\ddot{\boldsymbol{\theta}}_{d, j}=\ddot{\boldsymbol{\theta}}_{d}\left(t^{n}+c_{j} \Delta t\right)$

where $c_{j}(j=1, \ldots, s)$ represent the integration nodes of the $s$-stage Runge-Kutta method [20].

\section{Libration motion of flexible tethers}

The libration motion of flexible tethers of an E-sail is described in the coordinate system $O^{\prime} X_{b^{\prime}} Y_{b^{\prime}} Z_{b^{\prime}}$ as shown in Fig. 3. Different from the rigid tether or dumbbell model of tether, there will be $n$ sets of libration (in-plane and out-of-plane) angles for each tether if it is divided into $n$ elements $[33,35]$. This makes libration control difficult for the tether. To address this challenge, virtual in-plane and out-ofplane angles are introduced here by a virtual straight tether $\mathrm{AB}$ (red dot line) in Fig. 3, which connects the first and last nodes of each flexible tether. Thus, the libration motion of the tether is approximated by the
Table 1 Physical parameters of the E-sail system

\begin{tabular}{ll}
\hline Parameters & Values \\
\hline Mass of central spacecraft $(\mathrm{kg})$ & 400 \\
Shape of central spacecraft & Cylinder \\
Height of central spacecraft (m) & 2.0 \\
Radius of central spacecraft (m) & 2.0 \\
Initial orbit of E-sail & Circle \\
Position of central spacecraft $O_{b}(\mathrm{AU})$ & 1.0 \\
Number of tethers of E-sail & 4 \\
Density of tether material $\left(\mathrm{kg} / \mathrm{m}^{3}\right)$ & 1440.0 \\
Diameter of tether (m) & $7.38 \times 10^{-5}$ \\
Elastic module of tether $(\mathrm{GPa})$ & 70.0 \\
Initial location in solar system $(\mathrm{AU})$ & $(1,0,0)$ \\
Initial length of each tether (m) & 10.0 \\
Initial angular velocity of central spacecraft $(\mathrm{deg} / \mathrm{s}) \omega^{X_{b}}$ & $1.141 \times 10^{-5}$ \\
Initial angular velocity of central spacecraft $(\mathrm{deg} / \mathrm{s}) \omega^{Z_{b}}$ & 0.48 \\
\hline
\end{tabular}


Table 3 Parameters of numerical simulation cases

\begin{tabular}{|c|c|c|c|c|}
\hline $\begin{array}{l}\text { Case } \\
\text { name }\end{array}$ & $\begin{array}{l}\text { Deployment velocity } \\
(\mathrm{m} / \mathrm{s})\end{array}$ & $\begin{array}{l}\text { Thrust at remote unit } \\
\text { (N) }\end{array}$ & $\begin{array}{l}\text { High-order flexural modes of tether included } \\
\text { (yes/no) }\end{array}$ & $\begin{array}{l}\text { Tether } 1 \text { jammed (after } \\
\left.100 \mathrm{~s}^{*}\right)\end{array}$ \\
\hline Case A & 0.00 & 0.000 & No (one element per tether) & No \\
\hline Case B & 0.20 & 0.000 & No (one element per tether) & No \\
\hline Case $\mathrm{C}$ & 0.20 & 0.000 & Yes (multiple elements per tether) & No \\
\hline Case D & 0.20 & 0.005 & No (one element per tether) & No \\
\hline Case E & 0.20 & 0.005 & Yes (multiple elements per tether) & No \\
\hline Case $\mathrm{F}$ & 0.10 & 0.005 & Yes (multiple elements per tether) & No \\
\hline Case $\mathrm{G}$ & 0.40 & 0.005 & Yes (multiple elements per tether) & No \\
\hline Case $\mathrm{H}$ & 0.20 & 0.010 & Yes (multiple elements per tether) & No \\
\hline Case I & 0.20 & 0.002 & Yes (multiple elements per tether) & No \\
\hline Case $\mathbf{J}$ & 0.20 & 0.005 & Yes (multiple elements per tether) & Yes \\
\hline
\end{tabular}

$* 100 \mathrm{~s}$ is chosen arbitrayly

libration motion of the virtual tether $\mathrm{AB}$ in terms of inplane (in-plane angle, rotating around the $\boldsymbol{Z}^{\prime}{ }_{b}$ axis) and out-of-plane angle (rotating around the $\boldsymbol{Y}^{\prime \prime}{ }_{b}$ that is the $\boldsymbol{Y}_{\boldsymbol{b}}{ }_{\boldsymbol{b}}$ axis after the first rotation), that is,

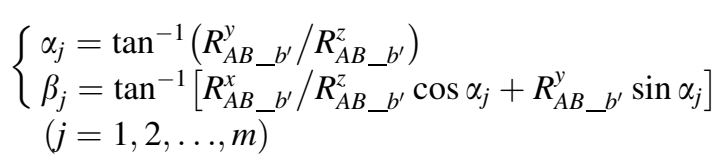
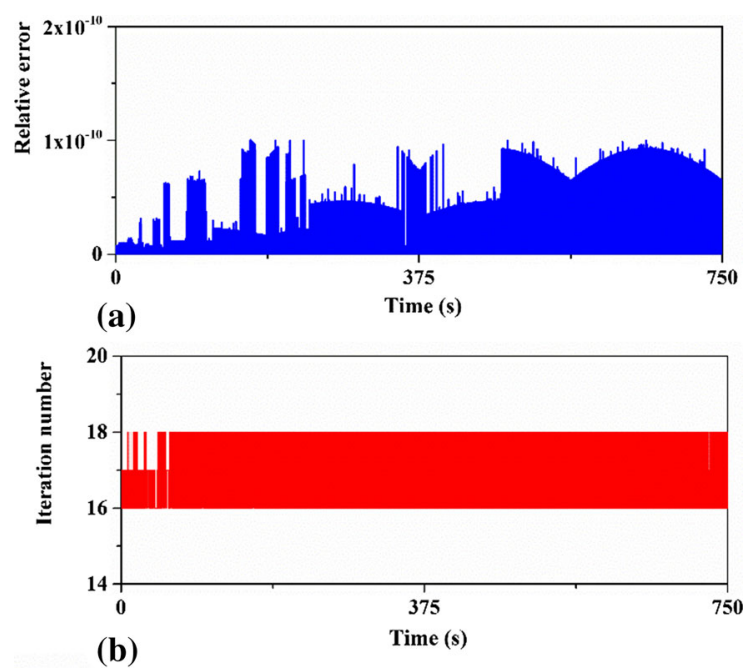

Fig. 4 Relative error and iteration numbers $\mathbf{a} \varepsilon$, b iteration number in Case A

$$
\begin{aligned}
&\left\{\begin{array}{l}
\boldsymbol{R}_{A B \_b^{\prime}, j}=\boldsymbol{T}_{b 2 b^{\prime}}\left(\boldsymbol{\theta}_{1}\right)_{j} \boldsymbol{T}_{g 2 b}(\boldsymbol{\theta}) \boldsymbol{R}_{A B \_g, j} \\
R_{A B \_g, j}=\left(X_{B \_g, j}-X_{A \_g, j}, Y_{B \_g, j}-Y_{A \_g, j}, Z_{B \_g, j}-Z_{A \_g, j}\right)^{T}
\end{array}\right. \\
&(j=1,2, \ldots, m)
\end{aligned}
$$

where $m$ represents the total number of tether of an Esail, and $\boldsymbol{R}_{A B_{-} b^{\prime}, j}=\left(\boldsymbol{R}_{A B_{-} b^{\prime}, j}^{x}, \boldsymbol{R}_{A B_{-} b^{\prime}, j}^{y}, \boldsymbol{R}_{A B_{-} b^{\prime}, j}^{z}\right)^{\mathrm{T}}$ is the vector of line $\mathrm{AB}$ expressed in the coordinate system $O^{\prime} X_{b^{\prime}} Y_{b^{\prime}} Z_{b^{\prime}}$, and $\boldsymbol{T}_{g 2 b}(\boldsymbol{\theta})=\boldsymbol{T}_{b 2 g}^{\mathrm{T}}(\boldsymbol{\theta})$. In addition, $\boldsymbol{T}_{b 2 b^{\prime}}\left(\boldsymbol{\theta}_{1}\right)_{j}$ is the transformation matrix from the coordinate system at $\mathrm{CM} O_{b} X_{b} Y_{b} Z_{b}$ to the coordinate system with the origin of the coordinate system $\left(O^{\prime} X_{b^{\prime}} Y_{b^{\prime}} Z_{b^{\prime}}\right)_{j}(j=1,2, \ldots, m)$ at the anchor point of each tether as shown in Fig. 3,

$\boldsymbol{T}_{b 2 b^{\prime}}\left(\boldsymbol{\theta}_{1}\right)=\left[\begin{array}{ccc}c \beta^{\prime} & c \alpha s \beta^{\prime} & s \alpha^{\prime} s \beta^{\prime} \\ s \beta^{\prime} & c \alpha^{\prime} c \beta^{\prime} & s \alpha^{\prime} c \beta^{\prime} \\ 0 & -s \alpha^{\prime} & c \beta^{\prime}\end{array}\right]$

where $\boldsymbol{X}_{\text {anc }, j}^{b}=\left(X_{\text {anc }, j}^{b}, Y_{\text {anc }, j}^{b}, Z_{\text {anc }, j}^{b}\right)^{\mathrm{T}}$ is the position vector of the anchor point of the $j$ th tether, $\alpha^{\prime}=\cos ^{-1}\left(X_{\text {anc }, j}^{b} / Y_{\text {anc }, j}^{b}\right)$, and $\beta^{\prime}=\sin ^{-1}\left(Z_{\mathrm{anc}, j}^{b} / \sqrt{\left(X_{\mathrm{anc}, j}^{b}\right)^{2}+\left(Y_{\mathrm{anc}, j}^{b}\right)^{2}}\right)$.

\section{Simulation results and discussion}

The system parameters of the E-sail in the simulation are given in Table $1[6,30]$. The E-sail is initially 
Fig. 5 Angular velocity of central spacecraft $\mathbf{a} \omega_{x}, \mathbf{b} \omega_{y}$, c $\omega_{z}$ in Case $\mathrm{A}$
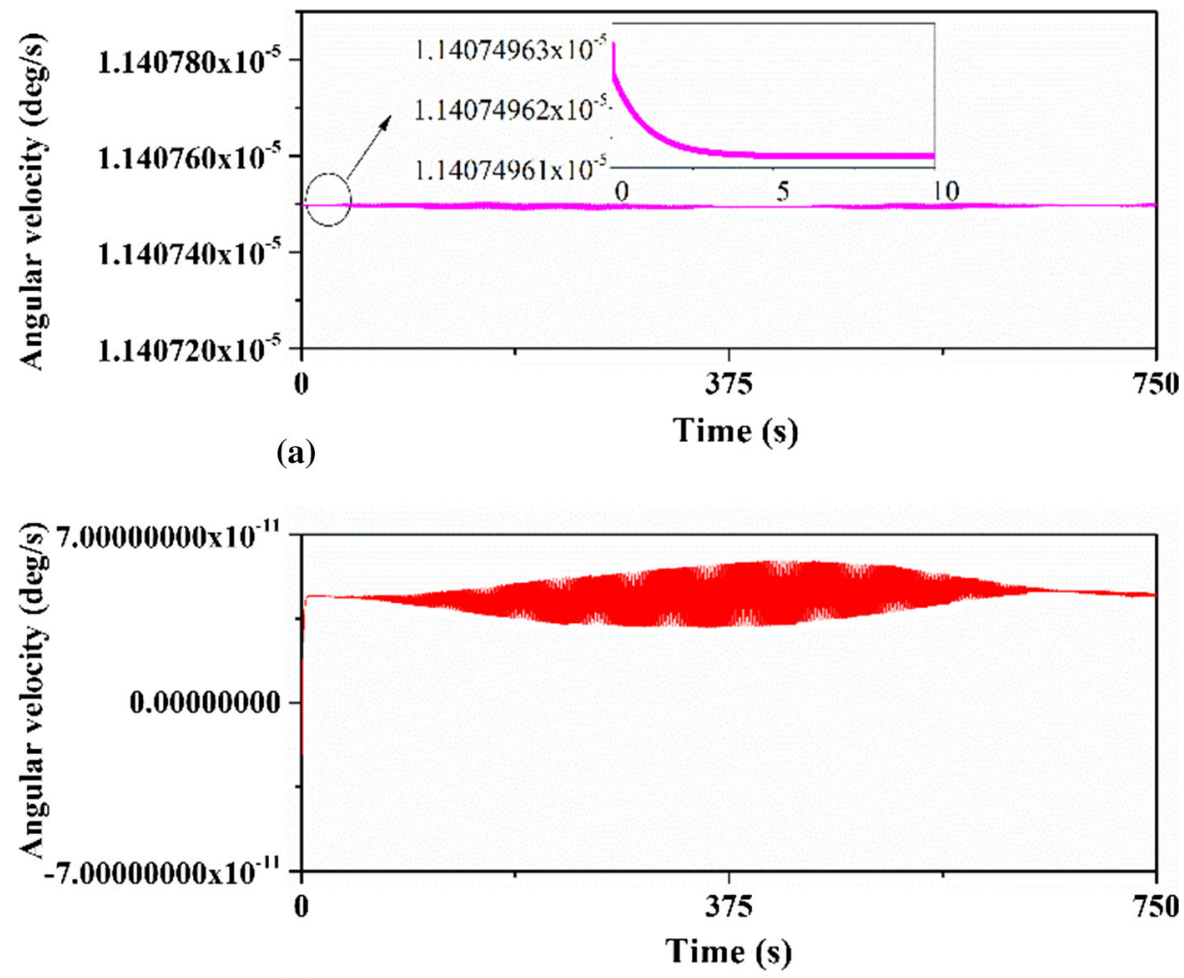

(b)

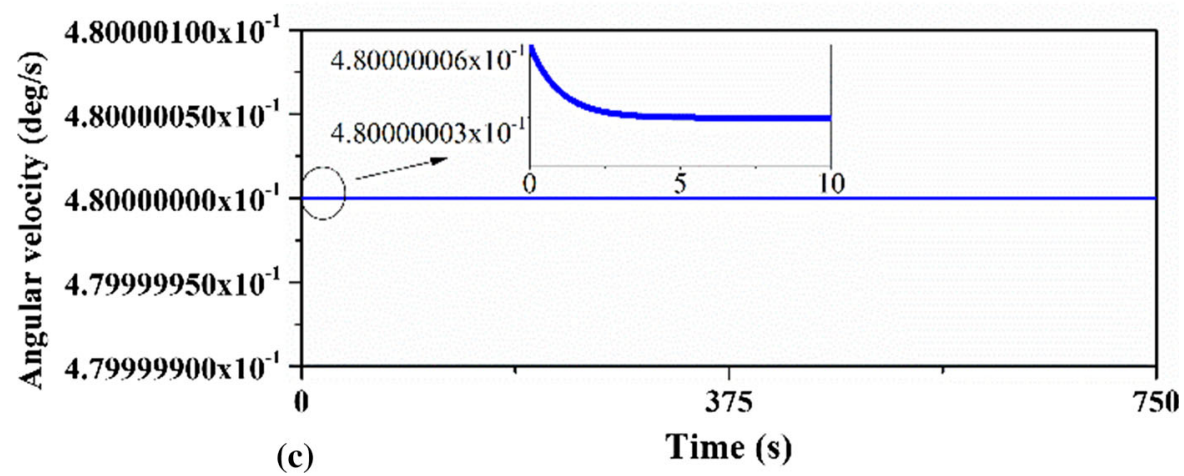

assumed in a circular orbit with orbital radius $R=1 \mathrm{AU}$. Its initial position is at $\left(X_{g}, Y_{g}, Z_{g}\right)=(1$ AU, 0,0$)$ in the global frame. It orbits in the positive direction of $O_{g} Z_{g}$ axis in the global frame with an orbital angular velocity, $\omega_{\text {orb }}=\omega^{X_{b}}=\sqrt{\mu_{S} / R^{3}}=$ $1.141 \times 10^{-5} \circ / \mathrm{s}$. Here, $\mu_{S}=1.3271244 \times 10^{20} \mathrm{~m}^{3} /$ $\mathrm{s}^{2}$ is the gravitational constant of the Sun. At the same time, the E-sail also spins about the $O_{b} Z_{b}$ axis in the positive direction at $0.48 \mathrm{deg} / \mathrm{s}$. Thus, the initial angular velocity vector of the central spacecraft is $\omega=\left(\omega^{X_{b}}, \omega^{Y_{b}}, \omega^{Z_{b}}\right)^{\mathrm{T}}=\left(1.141 \times 10^{-5}, 0,0.48\right)^{\mathrm{T}}$. The position vectors of the anchor points of tethers are listed in Table 2. Other perturbative forces except for the gravity of the Sun are not considered in the study. Besides, the two stages Gauss-Legendre Runge-Kutta with fourth-order accuracy is applied, and the Butcher table is listed in Eq. (40).

$$
\begin{array}{c|cc}
1 / 2-\sqrt{3} / 6 & 1 / 4 & 1 / 4-\sqrt{3} / 6 \\
1 / 2+\sqrt{3} / 6 & 1 / 4+\sqrt{3} / 6 & 1 / 4 \\
\hline 1 / 2+\sqrt{3} / 2 & 1 / 2-\sqrt{3} / 2
\end{array}
$$

Based on the trial and error, the iteration control parameters, $\varepsilon_{\max }$ and $q_{\max }$, are defined as $10^{-10}$ and 100 , respectively. The iteration will stop if either the 


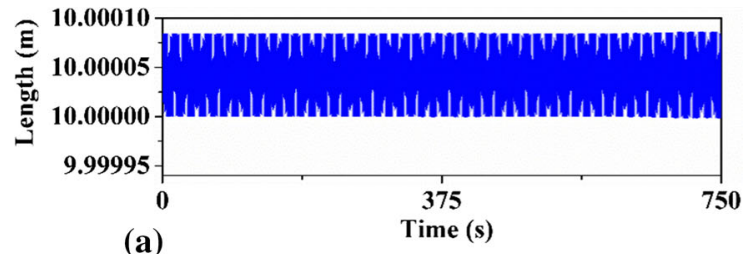

(a)

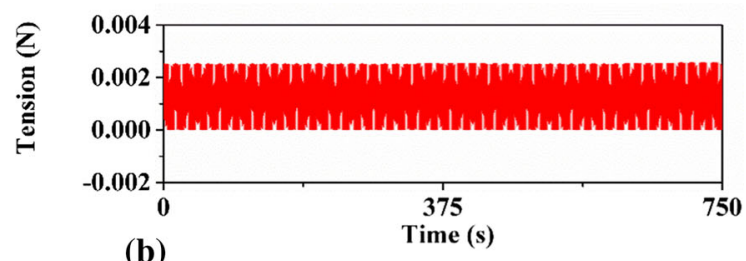

(b)

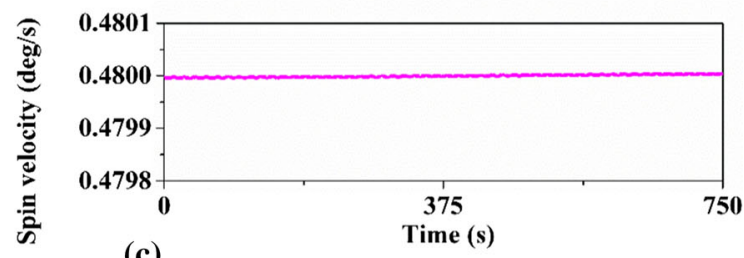

(c)

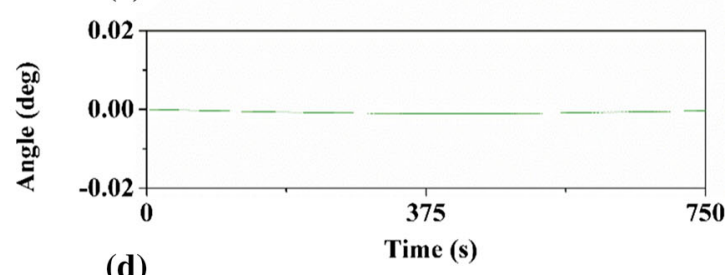

(d)

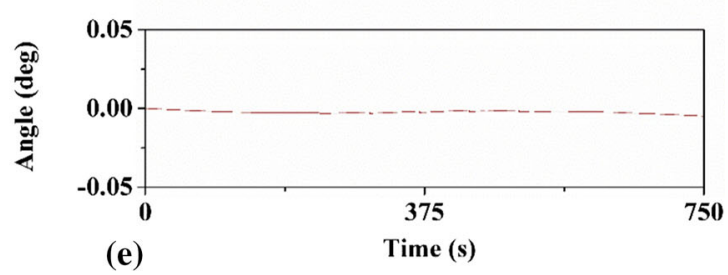

Fig. 6 Case A a tether length, b tether tension, c calculated angular velocity of remote unit (tether 1 ), $\mathbf{d}$ in-plane libration angle $\alpha_{1}$ of tether 1 , and $\mathbf{e}$ out-of-plane libration angle $\beta_{1}$ of tether 1

conditions $\varepsilon \leq \varepsilon_{\max }$ or $q \leq q_{\max }$ are satisfied. If both conditions are not satisfied, the simulation is deemed failed and stops. The unit of the central spacecraft's angular velocity is in $\mathrm{rad} / \mathrm{s}$ in the simulation and is presented as $\mathrm{deg} / \mathrm{s}$ in the results for reading convenience.

The initial conditions for the tethers and remote units are calculated by a special process by assuming the same angular velocities of the remote units and the central spacecraft initially about the $\boldsymbol{O}_{b} \boldsymbol{Z}_{b}$ axis direction. The detailed process can be found in the authors' work in [6].

\subsection{Validation of proposed method}

The proposed method is first validated by simulating an E-sail with constant-length tethers (deployed speed is zero), which is the Case A in Table 3. The PD controller is applied to stabilize the central spacecraft's attitude to avoid the loss of attitude stability of the central spacecraft caused by the oscillation of the tether [34]. The detailed implementation of the PD controller for the central spacecraft's attitude in the implicit Runge-Kutta integrator can be found in Section IV. The diagonal terms of the gain matrices $\boldsymbol{K}_{P}$ and $\boldsymbol{K}_{D}$ are chosen as $10^{6}$. The desired angular velocity of the central spacecraft is $\omega_{d}=$ $\left(1.141 \times 10^{-5}, 0,0.48\right)^{\mathrm{T}} \mathrm{deg} / \mathrm{s}$. For simplicity, each tether is modeled by one element only in the validation case, and the time step size is $0.0005 \mathrm{~s}$. The total simulation period is $750 \mathrm{~s}$.

The results of residual error $\varepsilon$ and the iteration number $m$ over the time are shown in Fig. 4, where the maximum iteration number never exceeds 18 , much less than the maximum allowed iteration number $m_{\max }=100$, while the residual error is controlled below $10^{-10}$ successfully. This indicates the proposed implicit $s$-stage Runge-Kutta time integrator works well. Figure 5 shows that the PD controller works successfully to control the central spacecraft spinning along the $\boldsymbol{O}_{b} \boldsymbol{Z}_{b}$-axis with a constant rate of $0.48 \mathrm{deg} / \mathrm{s}$. It also shows the large gains in the PD controller are necessary to decrease the response time of the E-sail, see in Fig. 5a and c. As expected, the angular velocity of remote units is in phase with that of the central spacecraft if no tether is deployed, see Fig. 6. For example, Fig. $6 \mathrm{c}$ shows the calculated angular velocity of the remote unit in the $\boldsymbol{O}_{b} \boldsymbol{Z}_{b}$ axis is equal to the spinning rate of the central spacecraft in the $O_{b} Z_{b}$ axis. The tether experiences a low tension, which suggests a heavy remote unit may be needed to increase the tether's tension resulting from the centrifugal effect for stability if the tether is getting slack [30]. Also, as shown in Fig. 6d and e, the tether's libration angles are small in out-of-plane without tether deployment, which is expected. In conclusion, the proposed highfidelity model of E-sail with the PD controller is validated for the central spacecraft's attitude control. 
Fig. 7 Snap shots of geometrical configuration of E-sail in Cases B and C

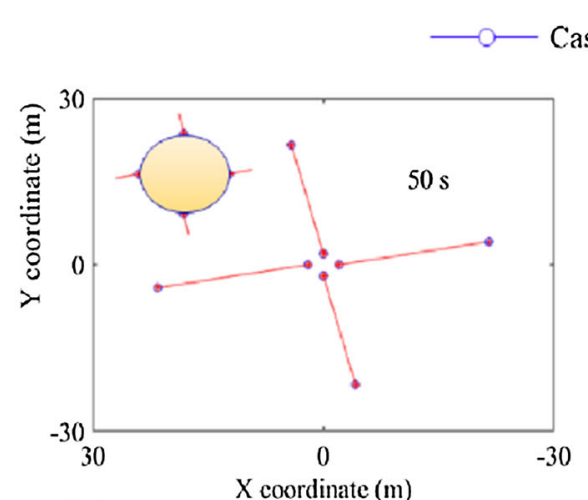

(a)

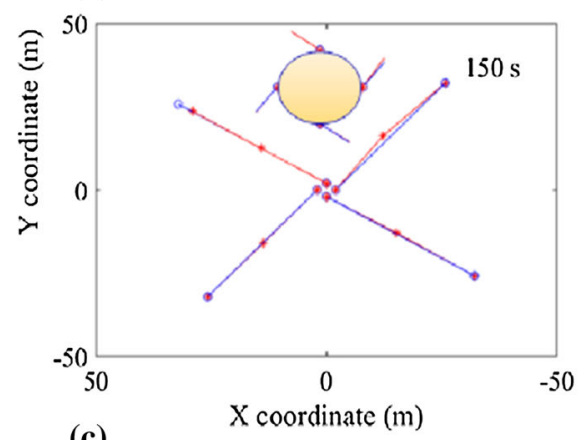

(c)
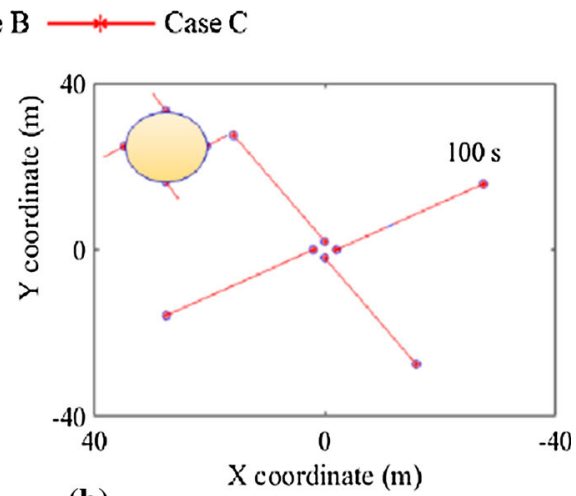

(b)

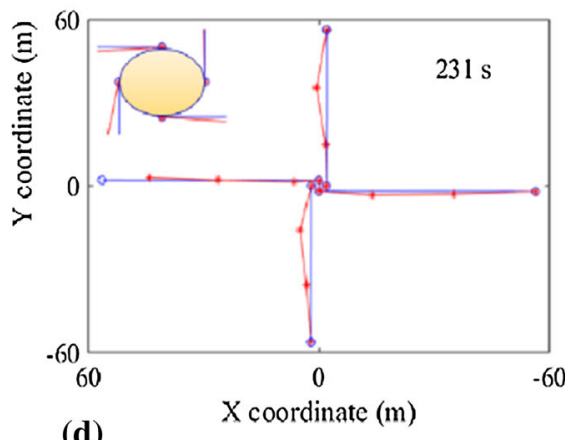

(d)
The kinematic constraints for the coupling of the flexible tether and the rigid body of central spacecraft work very well.

\subsection{Tether deployment without thrust at remote units}

Two numerical simulation cases (B and $\mathrm{C}$ listed in Table 3) are conducted to examine the effect of the tether's transverse flexural motion in the deployment process. The tethers are modeled with one element only in Case B and multiple elements in Case C. In Case $\mathrm{C}$, the process of the dividing of an element is activated when the tether deployment causes the length of the variable-length element longer than the upper bound of the elemental length $\left(L_{\max }\right)$. Based on the trial and error, the standard element length and upper bound $L_{\max }$ are set as $L_{s}=20 \mathrm{~m}$ and $L_{\max }=1.65 L_{\mathrm{s}}$.

Each tether's deployment speed is assumed the same as $0.2 \mathrm{~m} / \mathrm{s}$ for all tethers in both cases. The time step size and total simulation time are $0.0005 \mathrm{~s}$ and $750 \mathrm{~s}$, respectively. The simulation terminates when the libration angles (in-plane and out-of-plane angles) exceed $90^{\circ}$, which implies the tether wraps around the central spacecraft leading to the failure of tether deployment.

The simulation results are shown in Figs. 7 and 8. Figure 7 shows the snapshots of the tether configurations in the deployment process. The tethers start to bend immediately after deployment due to the Coriolis forces acting on the deployed tethers and remote units. It is because the tether does not have the bending rigidity to resist the bending moment caused by the Coriolis forces. However, the Coriolis forces are counter affected by the centrifugal forces on the same bodies to form a dynamic balance, which prevents the tether from immediately wrapping around the central spacecraft. When the length of the deployed tether is short, say less than or equal to the upper bound of the elemental length $L_{\max }$, the results of these two cases are the same. After the length of deployed tether is greater than the $L_{\max }$, a new element is generated and added to the tether discretization. The model in case $\mathrm{C}$ shows the flexural mode of the tether, which cannot be captured in case B with a single element as expected. It shows the multiple elements are needed to capture the flexural modes in the deployment dynamics of tether. The simulation stops at $231 \mathrm{~s}$ when the tether wraps around the central spacecraft. The corresponding 


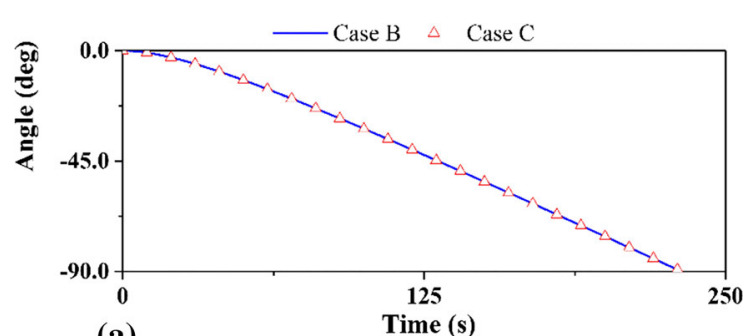

(a)

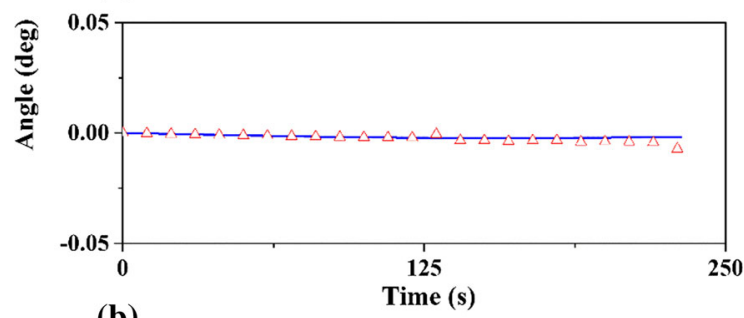

(b)

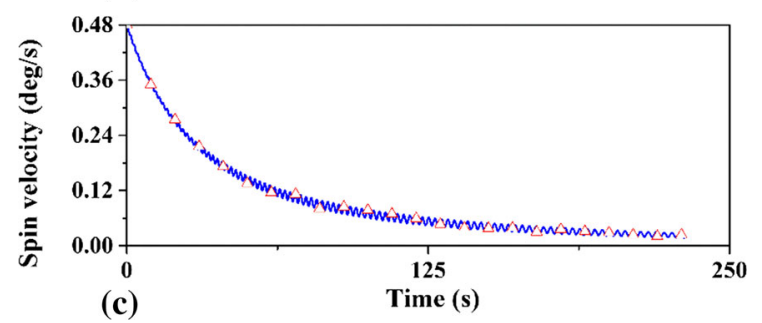

Fig. 8 Results of cases B and C (a) in-plane libration angle $\alpha_{1}$, b out-of-plane libration angle $\beta_{1}$, $\mathbf{c}$ spin velocity of remote unit (tether 1)

deployed tether length is $46.2 \mathrm{~m}$, which shows that the tether deployment fails.

Next, Fig. 8 shows the in-plane and out-of-plane libration angles and the calculated spin velocity of remote units relative to the central spacecraft. It is noted that the results of one and multiple elements models of the tether are remarkably close. Figure $8 a, b$ shows that the in-plane libration is the dominated libration mode. Two reasons can be attributed: (1) the Coriolis and centrifugal forces are in the plane of rotation, and (2) the perturbative forces that generate the out-of-plane component force are not considered in the current study. As a result, the tethers' out-ofplane libration angles will not be plotted in the following analysis because it is negligible when the other perturbative forces are not considered. Figure $8 \mathrm{a}$ and $\mathrm{c}$ show the in-plane angle of the first tether, and the spin velocity of the tip node of tether gradually increases and decreases, respectively, as the tether is deployed. It illustrates the remote units cannot keep in phase with the central spacecraft because the Coriolis force slows down the spin velocity of the remote units in the tether deployment process. The moment of Coriolis force increases as the tether length increases, which leads to tethers wrapping around the central spacecraft eventually. The results indicate that the external force, such as thrust at the remote unit, is needed to cancel the Coriolis effect to ensure successful tether deployment [36].

\subsection{Tether deployment with thrust at remote unit}

A tangential thrust is applied at the remote unit to avoid the tether wrapping of the central spacecraft. The direction of the thrust is along the opposite direction of the Coriolis force in the spin plane. A body-fixed frame $O_{B} \boldsymbol{X}_{b}^{\prime \prime \prime} \boldsymbol{Y}_{b}^{\prime \prime} \boldsymbol{Z}^{\prime \prime}{ }_{b}$ is introduced at the location of the remote units to implement the application of thrust. As shown in Fig. 3, the body-fixed frame $O_{B} \boldsymbol{X}_{b}^{\prime \prime \prime} \boldsymbol{Y}_{b}{ }_{b} \boldsymbol{Z}^{\prime \prime}{ }_{b}$ is parallel with the body-fixed frame $O_{A} \boldsymbol{X}_{b}^{\prime \prime \prime} \boldsymbol{Y}_{b}{ }_{b} \boldsymbol{Z}^{\prime \prime}{ }_{b}$ and is attached at point A. The tangential thrust $(0.005 \mathrm{~N})$ is applied in the positive direction of the $O_{B} \boldsymbol{Y}^{\prime \prime}{ }_{b}$ axis at point $\mathrm{B}$. To examine the effect of the transverse flexural motion of tether in the deployment dynamics, two cases (D and E in Table 3 ) are considered. Here, the standard-length $L_{s}$ of case E is set as $50 \mathrm{~m}$ to reduce the computational loads. The standard element length and upper bound $L_{\max }$ are set as $L_{s}=20 \mathrm{~m}$ and $L_{\max }=1.65 L_{\mathrm{s}}$, which is the same as that in Case C. The results are shown in Figs. 9 and 10 and Table 4.

As shown in Fig. 9, the E-sail can deploy more tether with wrapping the central spacecraft when a constant tangential thrust is applied at the remote units. Refs. [5, 11] show that a typical E-sail with tether length is ranging from 10 to $25 \mathrm{~km}$. Based on the results of the deployed $160 \mathrm{~m}$ tether, it can be estimated that the tether will eventually wrap (in-plane libration angle reaches $90^{\circ}$ ) the central spacecraft in cases of D and E, see in Table 4. It is because the resultant Coriolis force from the deployed tether and remote unit increases continuously as the tether length increases. The moment of the Coriolis force will eventually exceed the counterbalance moment by the constant tangential thrust. For instance, the in-plane angle reaches $90^{\circ}$ when the tether is $7515 \mathrm{~m}$ long in the case $\mathrm{D}$ (single element per tether) and $9104 \mathrm{~m}$ long in the case $\mathrm{E}$ (multiple elements per tether). This is because the effective spin radius of the remote unit is 
Fig. 9 Snap shots of geometric configuration of E-sail in cases D and E

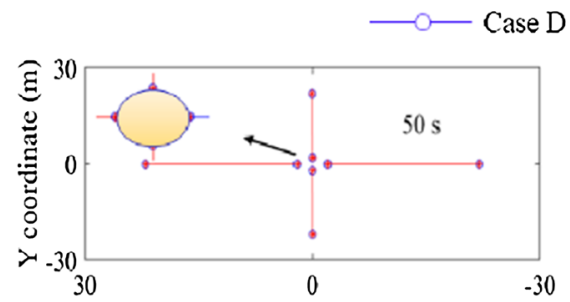

(a) $X$ coordinate $(\mathrm{m})$

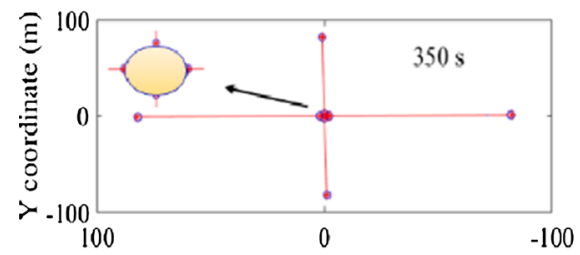

(c) $\quad X$ coordinate $(\mathrm{m})$

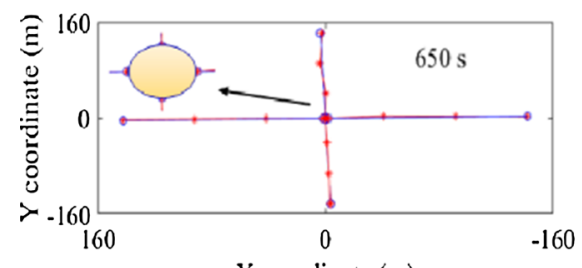

(e)

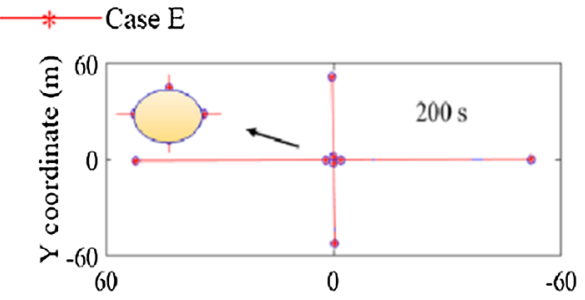

(b) $\quad X$ coordinate $(m)$

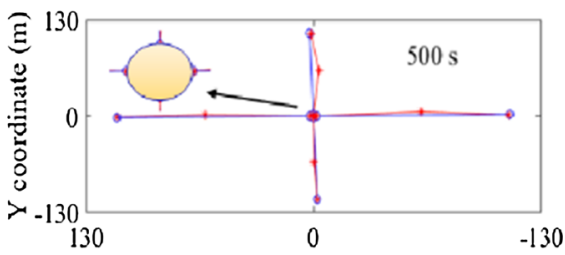

(d)

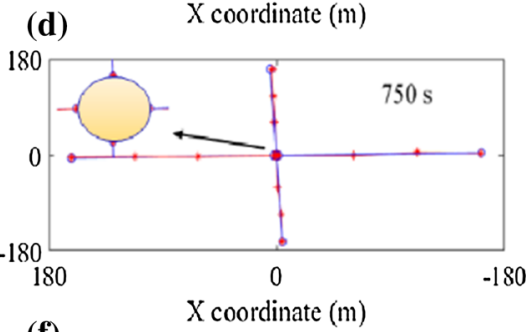

(f)

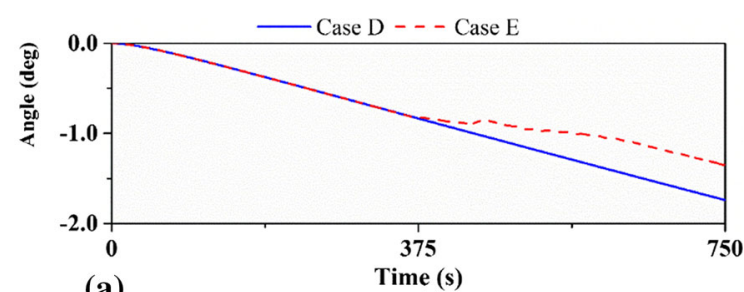

(a)

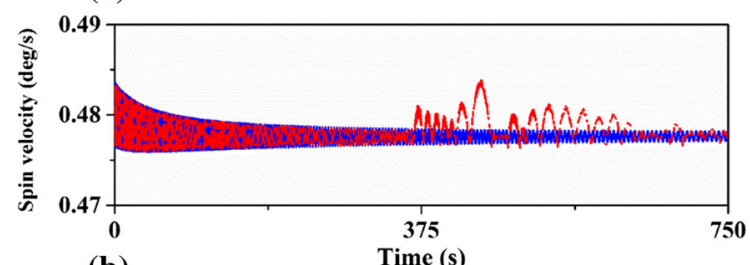

(b)

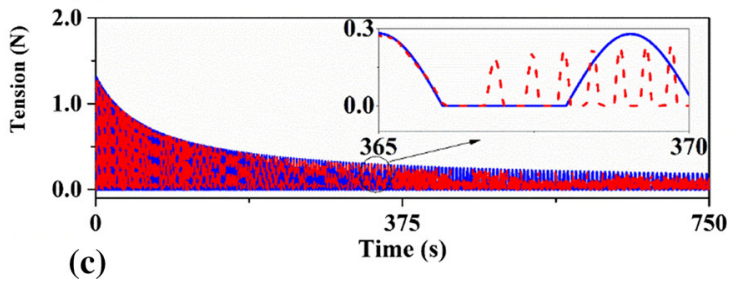

Fig. 10 Results of cases D and E (tether 1) a in-plane libration angle $\alpha_{1}$, b spin velocity of remote unit, and $\mathbf{c}$ tether tension at the remote unit shorter in the case E due to the tether's flexural bending than that in case $\mathrm{D}$ (one element per tether). It indicates the flexural mode of tether should be considered for precision analysis and control. Also, a radial thrust is needed to attenuate the wave oscillation along the tether [10].

The results of the in-plane libration angle, the spin velocity of the remote unit, and tether tension are shown in Fig. 10. Figure 10a shows the in-plane angles in cases $\mathrm{D}$ and $\mathrm{E}$ gradually increase and reach to $1.7^{\circ}$ and $1.4^{\circ}$ in the given period, respectively. The results of these two cases are close initially but eventually deviate due to the tether's flexural motion in case $\mathrm{E}$ becoming significant. Figure $10 \mathrm{~b}$ and $\mathrm{c}$ show the high-order modes in the case E due to the multiple elements per tether used in the analysis.

\subsection{Parametric study of the E-sail deployment}

To further investigate the effects of tether deployment speed and thrust magnitude on the E-sail's deployment dynamics, five numerical simulation cases $(\mathrm{E}-\mathrm{I})$ are conducted, see Table 3 . In all cases, the tethers are discretized into multiple elements per tether. The standard-length $L_{S}$, upper bound $L_{\max }$, and time step 
Table 4 Deployed length versus libration angle (average value of four tethers)

\begin{tabular}{llllll}
\hline Deployed length $(\mathrm{m})$ & 110 & 130 & 160 & 1600 (estimation) & 7515 (estimation) \\
Case D libration $\left({ }^{\circ}\right)$ & -1.14 & -1.38 & -1.74 & -19.00 & -90.00 \\
Deployed length $(\mathrm{m})$ & 110 & 130 & 160 & 1600 (estimation) & 9104 (estimation) \\
Case E libration $\left({ }^{\circ}\right)$ & -0.93 & -1.16 & -1.39 & -15.79 & -90.00 \\
\hline
\end{tabular}

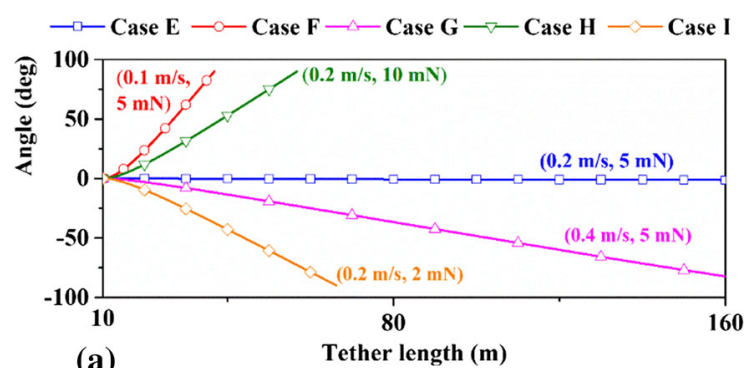

(a)

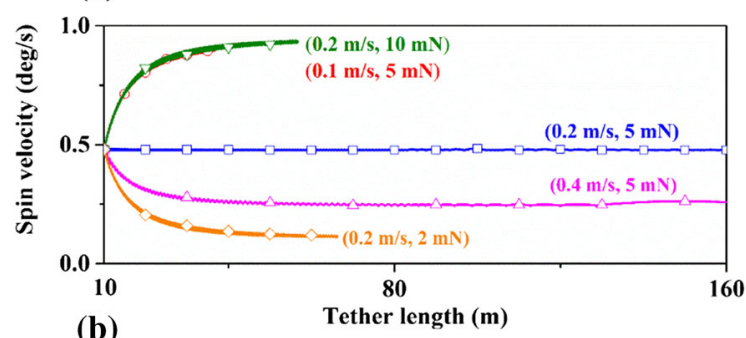

(b)

Fig. 11 Results of parametric analysis a in-plane libration angle $\alpha_{1}$, b spin velocity of remote unit (tether 1 )

size are set the same as those in Case D. In all cases, the magnitudes of the thrust are kept constant, such that $0.005 \mathrm{~N}$ in cases $(\mathrm{E}, \mathrm{F}, \mathrm{G}), 0.010 \mathrm{~N}$ in case $\mathrm{H}$, and $0.002 \mathrm{~N}$ in case $\mathrm{I}$.

Figure 11 shows the variation of the deployed tether length versus the in-plane libration angle and the spin velocity of the remote unit. The results reveal that a proper choice of the thrust and deployment speed is critical in suppressing the libration motion of flexible tethers to achieve the E-sail's successful deployment.

Figure 12 shows the tension of the variable-length element of the first tether. It illustrates that the tether is in the low-tension region, and the phenomenon of tether slack occurs. For example, Fig. 12a shows the variable-length element's tension varies between 0 and $0.2 \mathrm{~N}$ in the tether deployment process. It infers that the central spacecraft's spin rate controller should be applied to avoid the tether slack.
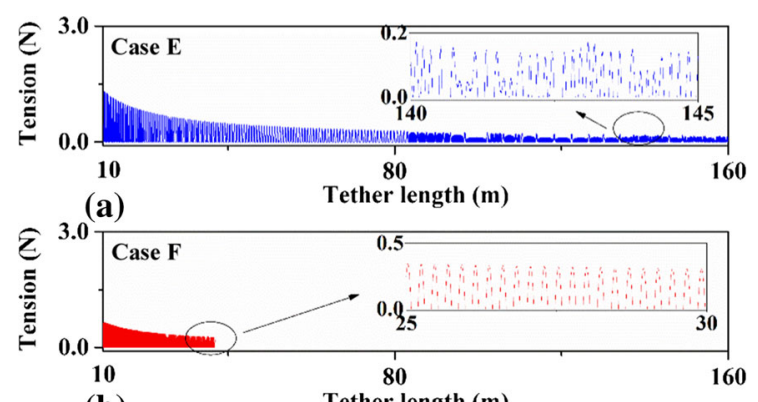

(b)

Tether length (m)
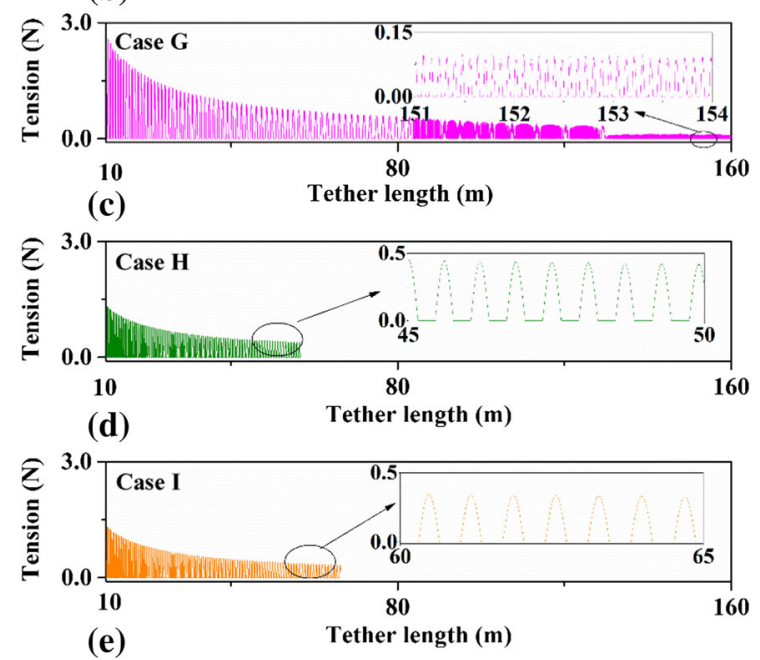

Fig. 12 Tether tension of the tether 1 at the central spacecraft

\subsection{Tether deployment with tether jammed}

As listed in Table 3, a numerical simulation case $\mathbf{J}$ is conducted. It is assumed that the deploying mechanism for tether one is jammed at $100 \mathrm{~s}$ after the central spacecraft starts to deploy tethers. The thrust on tether one is stopped accordingly. The other parameters in case $\mathrm{E}$ are assumed the same as those in case $\mathrm{E}$. The results are shown in Figs. 13 and 14.

Figure 13 shows the changes caused by the jammed tether in the libration angle. The spin velocity of the remote unit associated with the jammed and unjammed tethers varies significantly from the case 
Fig. 13 Results of cases E and $\mathbf{J}(\mathbf{a})$ in-plane libration angle $\alpha_{1}$, b spin velocity of remote unit (tether 1), c spin velocity of remote unit (tether 2), $\mathbf{d}$ tension at remote unit (tether 1), e tension at remote unit (tether 2)

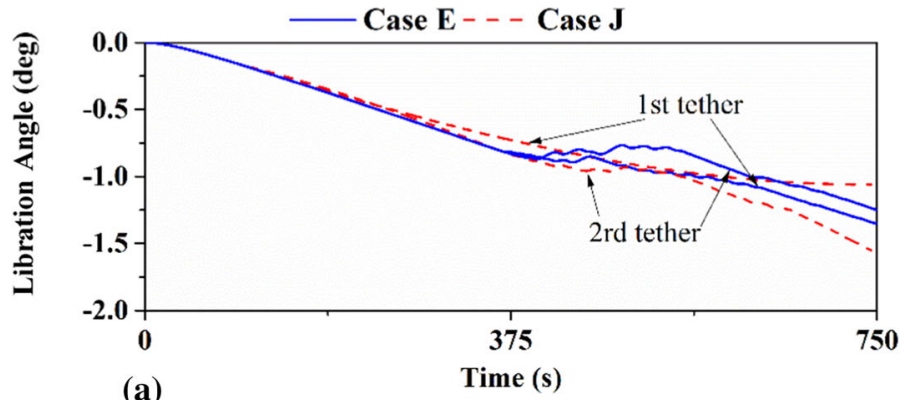

(a)

Time (s)

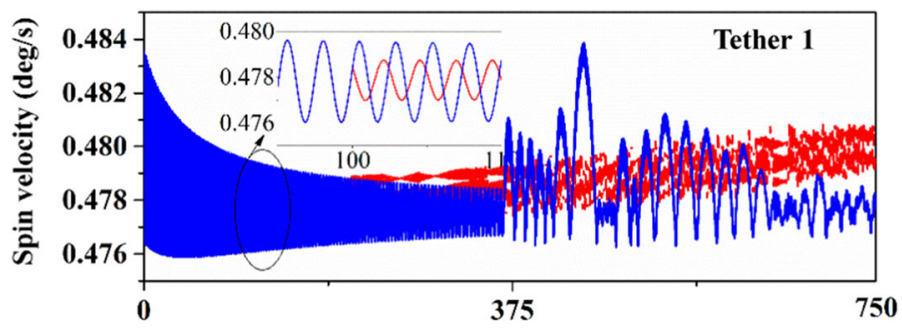

(b)

Time (s)
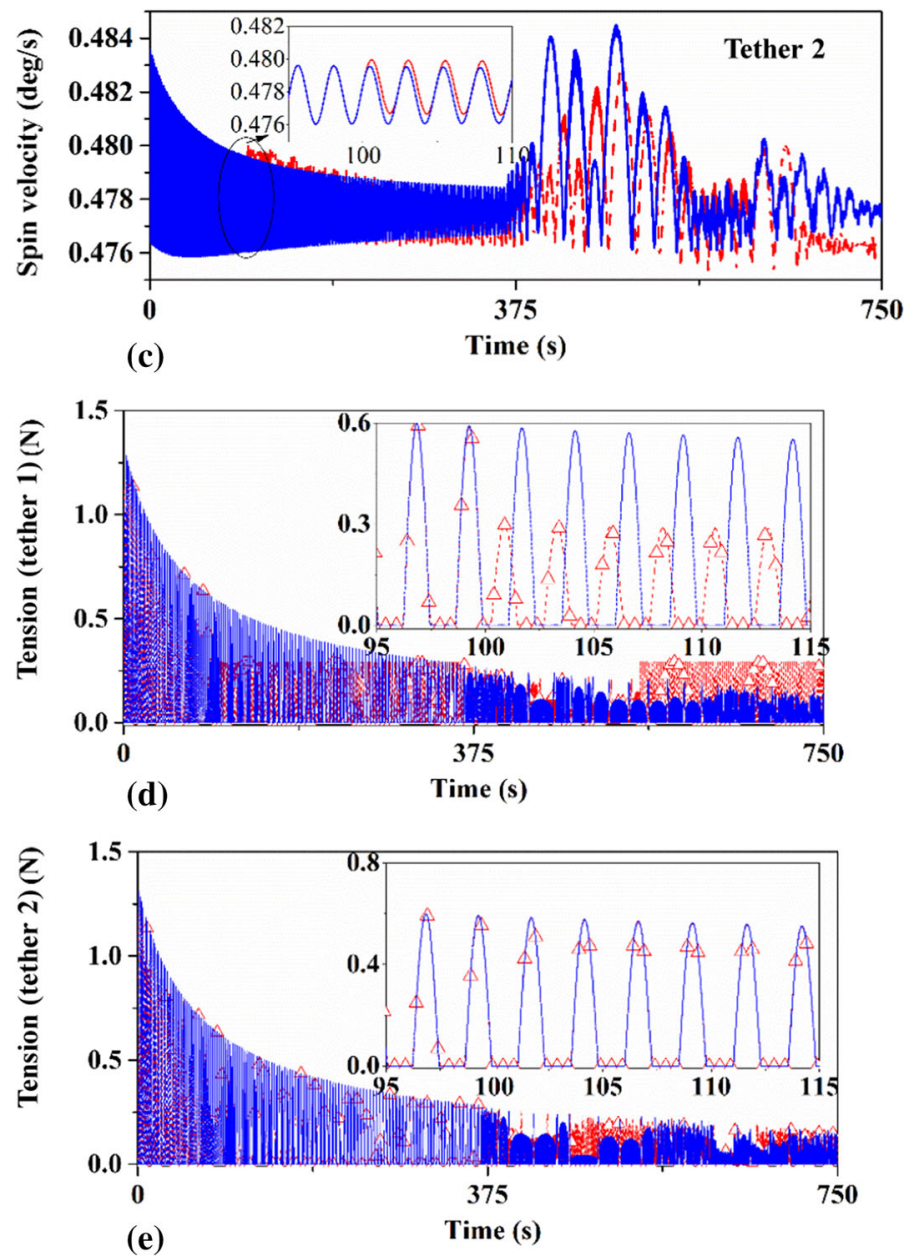
Fig. 14 Geometric configuration of E-sail in cases $\mathrm{E}$ and $\mathrm{J}$

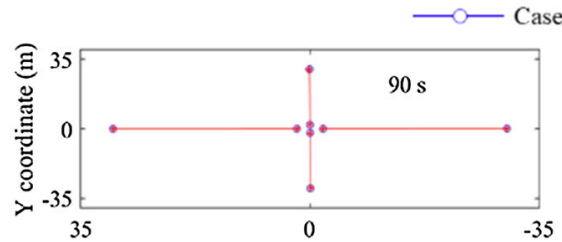

(a) $\mathrm{X}$ coordinate $(\mathrm{m})$

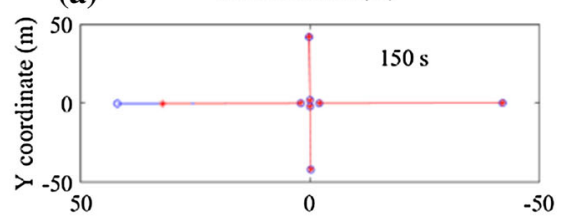

(c)

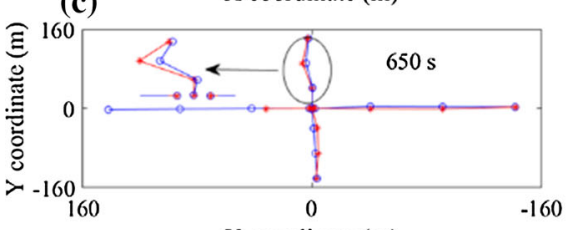

(e)
$\mathrm{X}$ coordinate $(\mathrm{m})$

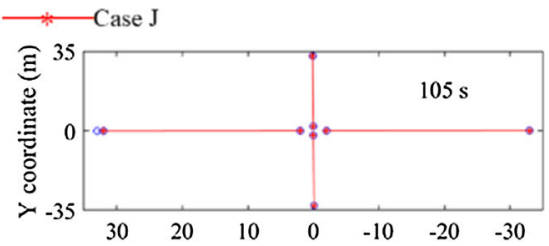

(b)

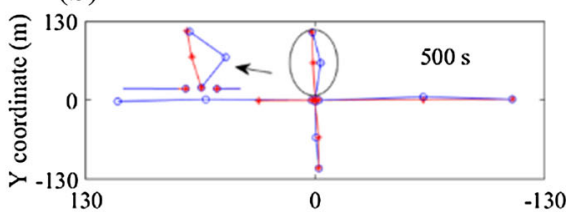

(d)

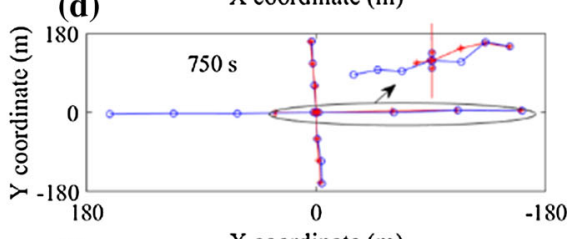

(f)

$\mathrm{X}$ coordinate $(\mathrm{m})$
E, where the tether is not jammed. For example, as shown in Fig. 13b and d, the spin velocity of the remote unit and tension associated tether one drops sharply when the tether is jammed. It is because the additional external forces, such as the Coriolis and thrust at the remote unit, disappears when the tether is jammed. As a result, the first tether's response in case $\mathbf{J}$ is quite different from the case $\mathrm{E}$, where the first tether one is not jammed. For the second tether, Fig. 13c and e shows the second tether's responses for the cases $\mathrm{E}$ and $\mathbf{J}$ are remarkably similar as expected because the second tether is not jammed. Finally, Fig. 14 shows the E-sail successfully deploys the targeted tether length for the rest three tethers. It shows the failure of a single tether could be isolated from the rest tethers when each tether has an isolated deployment mechanism, and the failure of tether deployment is isolated from other tethers. Therefore, the radial deployment method's deployment success rate is high because each tether has an isolated deployment mechanism.

\section{Conclusions}

In this study, the dynamics and control of radial tether deployment of a spinning E-sail are studied by a highfidelity three-dimensional model. In the model, the effects of flexible elastic tether dynamics, rigid-body dynamics of the central spacecraft, and the kinematic coupling between the flexible tethers and the rigidbody central spacecraft are included. Our results show that the proposed implicit Runge-Kutta integration scheme with a symplectic property can ensure the kinematic constraint conditions is precisely satisfied. The proportional-derivative controller is applied to stabilize the attitudes of the central spacecraft by rotating at a constant spin rate. The results show the E-sail cannot deploy long tethers without thrust at the remote units in the radial deployment mode. Tangential thrust is required at the remote units to counterbalance the Coriolis forces acting on the tethers and remote units to deploy tethers in the radial deployment mode successfully. Moreover, the parametric analysis shows the tether deployment speed and the thrust magnitude significantly affect deployment stability, which opens the possibility of successfully deploying tethers by advanced control strategies such as optimal control. Finally, the analysis shows the radial deployment is advantageous in isolating the failure of a single tether jammed in the deployment process from the successful deployment of the rest tethers.

Acknowledgements This work is supported by the Discovery Grant (RGPIN-2018-05991) and Discovery Accelerate Supplement Grant (RGPAS-2018-522709) of Natural Sciences and Engineering Research Council of Canada. 
Compliance with ethical standards

Conflict of interest The authors declare no conflict of interest in this article.

\section{Appendix: Expression of Jacobian matrix}

The Jacobian matrix $\boldsymbol{J}$ of Eq. (27) are as,

$$
\begin{aligned}
& \frac{\partial \boldsymbol{g}_{1}}{\partial \boldsymbol{K}_{1, \boldsymbol{X}_{s}}}=\boldsymbol{I}_{3 \times 3}, \frac{\partial \boldsymbol{g}_{1}}{\partial \boldsymbol{K}_{1, \boldsymbol{V}_{s}}}=-\Delta t a_{11} \boldsymbol{I}_{3 \times 3}, \quad \text { and } \\
& \frac{\partial \boldsymbol{g}_{1}}{\partial \boldsymbol{K}_{2, \boldsymbol{V}_{s}}}=-\Delta t a_{12} \boldsymbol{I}_{3 \times 3}
\end{aligned}
$$

$\frac{\partial \boldsymbol{g}_{2}}{\partial \boldsymbol{K}_{2, \boldsymbol{X}_{s}}}=\boldsymbol{I}_{3 \times 3}, \frac{\partial \boldsymbol{g}_{1}}{\partial \boldsymbol{K}_{2, \boldsymbol{V}_{s}}}=-\Delta t a_{21} \boldsymbol{I}_{3 \times 3}, \quad$ and

$$
\frac{\partial \boldsymbol{g}_{2}}{\partial \boldsymbol{K}_{2, \boldsymbol{V}_{s}}}=-\Delta t a_{22} \boldsymbol{I}_{3 \times 3}
$$

$\frac{\partial \boldsymbol{g}_{3}}{\partial \boldsymbol{K}_{1, \boldsymbol{V}_{s}}}=\boldsymbol{M}_{s}, \frac{\partial \boldsymbol{g}_{3}}{\partial \boldsymbol{K}_{1, \lambda_{1}}}=\Delta t a_{11} \boldsymbol{C}_{1, \boldsymbol{X}_{s}}^{\mathrm{T}}, \quad$ and

$$
\frac{\partial \boldsymbol{g}_{3}}{\partial \boldsymbol{K}_{2, \lambda_{1}}}=\Delta t a_{12} \boldsymbol{C}_{1, \boldsymbol{X}_{s}}^{\mathrm{T}}
$$

$\frac{\partial \boldsymbol{g}_{4}}{\partial \boldsymbol{K}_{2, \boldsymbol{V}_{s}}}=\boldsymbol{M}_{s}, \frac{\partial \boldsymbol{g}_{4}}{\partial \boldsymbol{K}_{1, \lambda_{1}}}=\Delta t a_{21} \boldsymbol{C}_{1, \boldsymbol{X}_{s}}^{\mathrm{T}}, \quad$ and

$$
\begin{aligned}
& \frac{\partial \boldsymbol{g}_{4}}{\partial \boldsymbol{K}_{2, \lambda_{1}}}=\Delta t a_{22} \boldsymbol{C}_{1, \boldsymbol{X}_{s}}^{\mathrm{T}} \\
& \frac{\partial \boldsymbol{g}_{5}}{\partial \boldsymbol{K}_{1, \boldsymbol{\theta}}}=\boldsymbol{I}_{3 \times 3}-\frac{\partial[\boldsymbol{J}(\underline{\boldsymbol{\theta}}) \underline{\boldsymbol{\omega}}]}{\partial \boldsymbol{K}_{1, \boldsymbol{\theta}}}, \frac{\partial \boldsymbol{g}_{5}}{\partial \boldsymbol{K}_{2, \boldsymbol{\theta}}}=-\frac{\partial[\boldsymbol{J}(\underline{\boldsymbol{\theta}}) \underline{\boldsymbol{\omega}}]}{\partial \boldsymbol{K}_{2, \boldsymbol{\theta}}} \\
& \text { and } \quad \underline{\boldsymbol{\theta}}=\boldsymbol{\theta}^{n}+\Delta t\left(a_{11} \boldsymbol{K}_{1, \boldsymbol{\theta}}+a_{12} \boldsymbol{K}_{2, \boldsymbol{\theta}}\right)
\end{aligned}
$$$$
\frac{\partial \boldsymbol{g}_{5}}{\partial \boldsymbol{K}_{1, \boldsymbol{\omega}}}=-\frac{\partial[\boldsymbol{J}(\underline{\boldsymbol{\theta}}) \underline{\boldsymbol{\omega}}]}{\partial \boldsymbol{K}_{1, \boldsymbol{\omega}}}, \frac{\partial \boldsymbol{g}_{5}}{\partial \boldsymbol{K}_{2, \boldsymbol{\omega}}}=-\frac{\partial[\boldsymbol{J}(\underline{\boldsymbol{\theta}}) \underline{\boldsymbol{\omega}}]}{\partial \boldsymbol{K}_{2, \boldsymbol{\omega}}},
$$$$
\text { and } \quad \underline{\omega}=\omega^{n}+\Delta t\left(a_{11} \boldsymbol{K}_{1, \boldsymbol{\omega}}+a_{12} \boldsymbol{K}_{2, \boldsymbol{\omega}}\right)
$$

$$
\begin{aligned}
\frac{\partial \boldsymbol{g}_{6}}{\partial \boldsymbol{K}_{1, \boldsymbol{\theta}}} & =-\frac{\partial[\boldsymbol{J}(\sim \boldsymbol{\theta}) \sim \boldsymbol{\omega}]}{\partial \boldsymbol{K}_{1, \boldsymbol{\theta}}}, \frac{\partial \boldsymbol{g}_{6}}{\partial \boldsymbol{K}_{2, \boldsymbol{\theta}}}=\boldsymbol{I}_{3 \times 3}-\frac{\partial[\boldsymbol{J}(\sim \boldsymbol{\theta}) \sim \boldsymbol{\omega}]}{\partial \boldsymbol{K}_{2, \boldsymbol{\theta}}}, \\
\text { and } & \sim \boldsymbol{\theta}=\boldsymbol{\theta}^{n}+\Delta t\left(a_{21} \boldsymbol{K}_{1, \boldsymbol{\theta}}+a_{22} \boldsymbol{K}_{2, \boldsymbol{\theta}}\right)
\end{aligned}
$$

$$
\begin{aligned}
\frac{\partial \boldsymbol{g}_{6}}{\partial \boldsymbol{K}_{1, \boldsymbol{\omega}}} & =-\frac{\partial[\boldsymbol{J}(\sim \boldsymbol{\theta}) \sim \boldsymbol{\omega}]}{\partial \boldsymbol{K}_{1, \boldsymbol{\omega}}}, \frac{\partial \boldsymbol{g}_{6}}{\partial \boldsymbol{K}_{2, \boldsymbol{\omega}}}=-\frac{\partial[\boldsymbol{J}(\sim \boldsymbol{\theta}) \sim \boldsymbol{\omega}]}{\partial \boldsymbol{K}_{2, \boldsymbol{\omega}}}, \\
\text { and } & \sim \boldsymbol{\omega}=\boldsymbol{\omega}^{n}+\Delta t\left(a_{21} \boldsymbol{K}_{1, \boldsymbol{\omega}}+a_{22} \boldsymbol{K}_{2, \boldsymbol{\omega}}\right)
\end{aligned}
$$

$$
\begin{gathered}
\frac{\partial \boldsymbol{g}_{7}}{\partial \boldsymbol{K}_{1, \boldsymbol{\omega}}}=\boldsymbol{H}_{s}+\frac{\partial\left[\underline{\boldsymbol{\omega}} \times\left(\boldsymbol{H}_{s} \underline{\boldsymbol{\omega}}\right)\right]}{\partial \boldsymbol{K}_{1, \boldsymbol{\omega}}}, \quad \text { and } \\
\frac{\partial \boldsymbol{g}_{7}}{\partial \boldsymbol{K}_{2, \boldsymbol{\omega}}}=\frac{\partial\left[\underline{\boldsymbol{\omega}} \times\left(\boldsymbol{H}_{s} \underline{\boldsymbol{\omega}}\right)\right]}{\partial \boldsymbol{K}_{2, \boldsymbol{\omega}}}
\end{gathered}
$$

$\frac{\partial \boldsymbol{g}_{7}}{\partial \boldsymbol{K}_{1, \lambda_{1}}}=\Delta t a_{11} \boldsymbol{C}_{1, \boldsymbol{\theta}}^{\mathrm{T}} \quad$ and $\quad \frac{\partial \boldsymbol{g}_{7}}{\partial \boldsymbol{K}_{2, \lambda_{1}}}=\Delta t a_{12} \boldsymbol{C}_{1, \boldsymbol{\theta}}^{\mathrm{T}}$

$$
\begin{gathered}
\frac{\partial \boldsymbol{g}_{8}}{\partial \boldsymbol{K}_{1, \boldsymbol{\omega}}}=\frac{\partial\left[\sim \boldsymbol{\omega} \times\left(\boldsymbol{H}_{s}^{\sim} \boldsymbol{\omega}\right)\right]}{\partial \boldsymbol{K}_{1, \boldsymbol{\omega}}}, \quad \text { and } \\
\frac{\partial \boldsymbol{g}_{8}}{\partial \boldsymbol{K}_{2, \boldsymbol{\omega}}}=\boldsymbol{H}_{s}+\frac{\partial\left[\sim \boldsymbol{\omega} \times\left(\boldsymbol{H}_{s} \sim \boldsymbol{\omega}\right)\right]}{\partial \boldsymbol{K}_{2, \boldsymbol{\omega}}}
\end{gathered}
$$

$\frac{\partial \boldsymbol{g}_{8}}{\partial \boldsymbol{K}_{1, \lambda_{1}}}=\Delta t a_{21} \boldsymbol{C}_{1, \boldsymbol{\theta}}^{\mathrm{T}} \quad$ and $\quad \frac{\partial \boldsymbol{g}_{8}}{\partial \boldsymbol{K}_{2, \lambda_{1}}}=\Delta t a_{22} \boldsymbol{C}_{1, \boldsymbol{\theta}}^{\mathrm{T}}$

$$
\begin{aligned}
& \frac{\partial \boldsymbol{g}_{9}}{\partial \boldsymbol{K}_{1, \boldsymbol{X}_{t}}}=\boldsymbol{I}_{4 m(n+1) \times 4 m(n+1)}, \\
& \frac{\partial \boldsymbol{g}_{9}}{\partial \boldsymbol{K}_{1, \boldsymbol{V}_{t}}}=-\Delta t a_{11} \boldsymbol{I}_{4 m(n+1) \times 4 m(n+1)},
\end{aligned}
$$

and $\frac{\partial \boldsymbol{g}_{9}}{\partial \boldsymbol{K}_{2, \boldsymbol{V}_{t}}}=-\Delta t a_{12} \boldsymbol{I}_{4 m(n+1) \times 4 m(n+1)}$

$$
\begin{aligned}
& \frac{\partial \boldsymbol{g}_{10}}{\partial \boldsymbol{K}_{2, \boldsymbol{X}_{t}}}=\boldsymbol{I}_{4 m(n+1) \times 4 m(n+1)}, \\
& \frac{\partial \boldsymbol{g}_{10}}{\partial \boldsymbol{K}_{1, \boldsymbol{V}_{t}}}=-\Delta t a_{21} \boldsymbol{I}_{4 m(n+1) \times 4 m(n+1)},
\end{aligned}
$$

$$
\text { and } \frac{\partial \boldsymbol{g}_{10}}{\partial \boldsymbol{K}_{2, \boldsymbol{V}_{t}}}=-\Delta t a_{22} \boldsymbol{I}_{4 m(n+1) \times 4 m(n+1)}
$$

$$
\begin{aligned}
& \frac{\partial \boldsymbol{g}_{11}}{\partial \boldsymbol{K}_{1, \boldsymbol{V}_{t}}}=\boldsymbol{M}_{t}, \frac{\partial \boldsymbol{g}_{11}}{\partial \boldsymbol{K}_{1, \lambda_{1}}}=\Delta t a_{11} \boldsymbol{C}_{1, \boldsymbol{X}_{t}}^{\mathrm{T}}, \quad \text { and } \\
& \frac{\partial \boldsymbol{g}_{11}}{\partial \boldsymbol{K}_{2, \lambda_{1}}}=\Delta t a_{12} \boldsymbol{C}_{1, \boldsymbol{X}_{t}}^{\mathrm{T}}
\end{aligned}
$$




$$
\begin{aligned}
& \frac{\partial \boldsymbol{g}_{11}}{\partial \boldsymbol{K}_{1, \lambda_{2}}}=\Delta t a_{11} \boldsymbol{C}_{2, \boldsymbol{X}_{t}}^{\mathrm{T}}, \frac{\partial \boldsymbol{g}_{11}}{\partial \boldsymbol{K}_{2, \boldsymbol{\lambda}_{2}}}=\Delta t a_{12} \boldsymbol{C}_{2, \boldsymbol{X}_{t}}^{\mathrm{T}}, \\
& \frac{\partial \boldsymbol{g}_{11}}{\partial \boldsymbol{K}_{1, \lambda_{3}}}=\Delta t a_{11} \boldsymbol{C}_{3, \boldsymbol{X}_{t}}^{\mathrm{T}}, \\
& \text { and } \frac{\partial \boldsymbol{g}_{11}}{\partial \boldsymbol{K}_{2, \lambda_{3}}}=\Delta t a_{12} \boldsymbol{C}_{3, \boldsymbol{X}_{t}}^{\mathrm{T}} \\
& \frac{\partial \boldsymbol{g}_{12}}{\partial \boldsymbol{K}_{2, \boldsymbol{V}_{t}}}=\boldsymbol{M}_{t}, \frac{\partial \boldsymbol{g}_{12}}{\partial \boldsymbol{K}_{1, \lambda_{1}}}=\Delta t a_{21} \boldsymbol{C}_{1, \boldsymbol{X}_{t}}^{\mathrm{T}}, \quad \text { and } \\
& \frac{\partial \boldsymbol{g}_{12}}{\partial \boldsymbol{K}_{2, \lambda_{1}}}=\Delta t a_{22} \boldsymbol{C}_{1, \boldsymbol{X}_{t}}^{\mathrm{T}} \\
& \frac{\partial \boldsymbol{g}_{12}}{\partial \boldsymbol{K}_{1, \lambda_{2}}}=\Delta t a_{21} \boldsymbol{C}_{2, \boldsymbol{X}_{t}}^{\mathrm{T}}, \frac{\partial \boldsymbol{g}_{12}}{\partial \boldsymbol{K}_{2, \boldsymbol{\lambda}_{2}}}=\Delta t a_{22} \boldsymbol{C}_{2, \boldsymbol{X}_{t}}^{\mathrm{T}}, \\
& \frac{\partial \boldsymbol{g}_{12}}{\partial \boldsymbol{K}_{1, \lambda_{3}}}=\Delta t a_{21} \boldsymbol{C}_{3, \boldsymbol{X}_{t}}^{\mathrm{T}}, \\
& \text { and } \frac{\partial \boldsymbol{g}_{12}}{\partial \boldsymbol{K}_{2, \lambda_{3}}}=\Delta t a_{22} \boldsymbol{C}_{3, \boldsymbol{X}_{t}}^{\mathrm{T}} \\
& \frac{\partial \boldsymbol{g}_{13}}{\partial \boldsymbol{K}_{1, \boldsymbol{X}_{s}}}=\Delta t a_{11} \boldsymbol{C}_{1, \boldsymbol{X}_{s}}, \frac{\partial \boldsymbol{g}_{13}}{\partial \boldsymbol{K}_{2, \boldsymbol{X}_{s}}}=\Delta t a_{12} \boldsymbol{C}_{1, \boldsymbol{X}_{s}}, \\
& \frac{\partial \boldsymbol{g}_{13}}{\partial \boldsymbol{K}_{1, \boldsymbol{\theta}}}=\Delta t a_{11} \boldsymbol{C}_{1, \boldsymbol{\theta}}, \\
& \frac{\partial \boldsymbol{g}_{13}}{\partial \boldsymbol{K}_{2, \boldsymbol{\theta}}}=\Delta t a_{12} \boldsymbol{C}_{1, \boldsymbol{\theta}}, \frac{\partial \boldsymbol{g}_{13}}{\partial \boldsymbol{K}_{1, \boldsymbol{X}_{s}}}=\Delta t a_{11} \boldsymbol{C}_{1, \boldsymbol{X}_{s}}, \\
& \text { and } \frac{\partial \boldsymbol{g}_{13}}{\partial \boldsymbol{K}_{2, \boldsymbol{X}_{s}}}=\Delta t a_{12} \boldsymbol{C}_{1, \boldsymbol{X}_{s}} \\
& \frac{\partial \boldsymbol{g}_{14}}{\partial \boldsymbol{K}_{1, \boldsymbol{X}_{s}}}=\Delta t a_{21} \boldsymbol{C}_{1, \boldsymbol{X}_{s}}, \frac{\partial \boldsymbol{g}_{14}}{\partial \boldsymbol{K}_{1, \boldsymbol{\theta}}}=\Delta t a_{21} \boldsymbol{C}_{1, \boldsymbol{\theta}}, \\
& \frac{\partial \boldsymbol{g}_{14}}{\partial \boldsymbol{K}_{1, \boldsymbol{X}_{s}}}=\Delta t a_{21} \boldsymbol{C}_{1, \boldsymbol{X}_{s}} \\
& \frac{\partial \boldsymbol{g}_{14}}{\partial \boldsymbol{K}_{2, \boldsymbol{X}_{s}}}=\Delta t a_{22} \boldsymbol{C}_{1, \boldsymbol{X}_{s}}, \frac{\partial \boldsymbol{g}_{14}}{\partial \boldsymbol{K}_{2, \boldsymbol{\theta}}}=\Delta t a_{22} \boldsymbol{C}_{1, \boldsymbol{\theta}}, \\
& \text { and } \frac{\partial \boldsymbol{g}_{14}}{\partial \boldsymbol{K}_{2, \boldsymbol{X}_{s}}}=\Delta t a_{22} \boldsymbol{C}_{1, \boldsymbol{X}_{s}} \\
& \frac{\partial \boldsymbol{g}_{15}}{\partial \boldsymbol{K}_{1, \boldsymbol{X}_{t}}}=\Delta t a_{11} \boldsymbol{C}_{2, \boldsymbol{X}_{s}}, \frac{\partial \boldsymbol{g}_{15}}{\partial \boldsymbol{K}_{2, \boldsymbol{X}_{t}}}=\Delta t a_{12} \boldsymbol{C}_{2, \boldsymbol{X}_{s}}, \\
& \frac{\partial \boldsymbol{g}_{16}}{\partial \boldsymbol{K}_{1, \boldsymbol{X}_{t}}}=\Delta t a_{21} \boldsymbol{C}_{2, \boldsymbol{X}_{s}}, \\
& \text { and } \frac{\partial \boldsymbol{g}_{16}}{\partial \boldsymbol{K}_{2, \boldsymbol{X}_{t}}}=\Delta t a_{22} \boldsymbol{C}_{2, \boldsymbol{X}_{s}}
\end{aligned}
$$

$$
\begin{aligned}
& \frac{\partial \boldsymbol{g}_{17}}{\partial \boldsymbol{K}_{1, \boldsymbol{X}_{t}}}=\Delta t a_{11} \boldsymbol{C}_{3, \boldsymbol{X}_{s}}, \frac{\partial \boldsymbol{g}_{17}}{\partial \boldsymbol{K}_{2, \boldsymbol{X}_{t}}}=\Delta t a_{12} \boldsymbol{C}_{3, \boldsymbol{X}_{s}}, \\
& \frac{\partial \boldsymbol{g}_{18}}{\partial \boldsymbol{K}_{1, \boldsymbol{X}_{t}}}=\Delta t a_{21} \boldsymbol{C}_{3, \boldsymbol{X}_{s}}, \\
& \text { and } \frac{\partial \boldsymbol{g}_{18}}{\partial \boldsymbol{K}_{2, \boldsymbol{X}_{t}}}=\Delta t a_{22} \boldsymbol{C}_{3, \boldsymbol{X}_{s}}
\end{aligned}
$$

\section{References}

1. Janhunen, P.: Electric sail for spacecraft propulsion. J. Propuls. Power 20(4), 763-764 (2004). https://doi.org/10. 2514/1.8580

2. Quarta, A.A., Mengali, G.: Electric sail mission analysis for outer solar system exploration. J. Guid. Control Dyn. 33(3), 740-755 (2010). https://doi.org/10.2514/1.47006

3. Bassetto, M., Quarta, A.A., Mengali, G.: Locally-optimal electric sail transfer. Proc. Inst. Mech. Eng. Part G J. Aerosp. Eng. 233(1), 166-179 (2019). https://doi.org/10. 1177/0954410017728975

4. Bassetto, M., Mengali, G., Quarta, A.A.: Stability and control of spinning electric solar wind sail in heliostationary orbit. J. Guid. Control Dyn. 42(2), 425-431 (2019). https:// doi.org/10.2514/1.g003788

5. Janhunen, P., Quarta, A., Mengali, G.: Electric solar wind sail mass budget model. Geosci. Instrum. Methods Data Syst. 2(1), 85-95 (2013). https://doi.org/10.5194/gi-2-852013

6. Li, G., Zhu, Z.H., Du, C., Meguid, S.A.: Characteristics of coupled orbital-attitude dynamics of flexible electric solar wind sail. Acta Astronaut. 159(June), 593-608 (2019). https://doi.org/10.1016/j.actaastro.2019.02.009

7. Mengali, G., Quarta, A.A., Janhunen, P.: Electric sail performance analysis. J. Spacecr. Rockets 45(1), 122-129 (2008). https://doi.org/10.2514/1.31769

8. Simmons, M., Montalvo, C.: Vibration modes of an electric sail. J. Guid. Control Dyn. 43(7), 1393-1398 (2020). https:// doi.org/10.2514/1.g004924

9. Liu, F., Hu, Q., Liu, Y.: Attitude dynamics of electric sail from multibody perspective. J. Guid. Control Dyn. 41(12), 2633-2646 (2018). https://doi.org/10.2514/1.g003625

10. Montalvo, C., Wiegmann, B.: Electric sail space flight dynamics and controls. Acta Astronaut. 148(July), 268-275 (2018). https://doi.org/10.1016/j.actaastro.2018.05.009

11. Fulton, J., Schaub, H.: Fixed-axis electric sail deployment dynamics analysis using hub-mounted momentum control. Acta Astronaut. 144(March), 160-170 (2018). https://doi. org/10.1016/j.actaastro.2017.11.048

12. Wang, R., Wei, C., Wu, Y., Zhao, Y., Cui, H.: Dynamic analysis of the spinning deployment for flexible tether electric sail spacecraft. J. Harbin Eng. Univ. 40(4), 724-729 (2019). https://doi.org/10.11990/jheu.201709101

13. Wang, R., Wei, C., Wu, Y., Zhao, Y.: The study of spin control of flexible electric sail using the absolute nodal coordinate formulation. In: 2017 IEEE International Conference on Cybernetics and Intelligent Systems and IEEE 
Conference on Robotics, Automation and Mechatronics, pp. 785-790 (2017). https://doi.org/10.1109/ICCIS.2017. 8274879

14. Tinker, M., Bryan, T., Vaughn, J., Canfield, S., Hargis, B., Hunter, J.D., McArthur, J.: Electric Sail Tether Deployment System for CubeSats, pp. 1-7 (2019). https://ntrs.nasa.gov/ citations/20190002121

15. Sun, G., Zhu, Z.H.: Fractional-order tension control law for deployment of space tether system. J. Guid. Control Dyn. 37(6), 2057-2062 (2014). https://doi.org/10.2514/1. g000496

16. Murugathasan, L., Zhu, Z.H.: Deployment control of tethered space systems with explicit velocity constraint and invariance principle. Acta Astronaut. 157(April), 390-396 (2019). https://doi.org/10.1016/j.actaastro.2019.01.017

17. Wang, C., Wang, P., Li, A., Guo, Y.: Deployment of tethered satellites in low-eccentricity orbits using adaptive sliding mode control. J. Aerosp. Eng. 30(6), 1-9 (2017). https://doi.org/10.1061/(ASCE)AS.1943-5525.0000793

18. Mantellato, R., Valmorbida, A., Lorenzini, E.C.: Thrustaided librating deployment of tape tethers. J. Spacecr. Rockets 52(5), 1395-1406 (2015). https://doi.org/10.2514/ 1.a33273

19. Boni, L., Bassetto, M., Mengali, G., Quarta, A.A.: Electric sail static structural analysis with finite element approach. Acta Astronaut. 175(October), 510-516 (2020). https://doi. org/10.1016/j.actaastro.2020.06.009

20. Li, G.Q., Zhu, Z.H.: Long-term dynamic modeling of tethered spacecraft using nodal position finite element method and symplectic integration. Celest. Mech. Dyn. Astron. 123, 363-386 (2015). https://doi.org/10.1007/s10569-015-96405

21. Li, G., Zhu, Z.H.: On libration suppression of partial space elevator with a moving climber. Nonlinear Dyn. 97(4), 2107-2125 (2019). https://doi.org/10.1007/s11071-01905108-0

22. Du, J., Cui, C., Bao, H., Qiu, Y.: Dynamic analysis of cabledriven parallel manipulators using a variable length finite element. J. Comput. Nonlinear Dyn. 10(1), 011013-011017 (2014). https://doi.org/10.1115/1.4026570

23. Yu, B., Jin, D.: Deployment and retrieval of tethered satellite system under $\mathbf{J}_{2}$ perturbation and heating effect. Acta Astronaut. 67(7), 845-853 (2010). https://doi.org/10. 1016/j.actaastro.2010.05.013

24. Tang, J., Ren, G., Zhu, W., Ren, H.: Dynamics of variablelength tethers with application to tethered satellite deployment. Commun. Nonlinear Sci. Numer. Simul. 16(8), 3411-3424 (2011). https://doi.org/10.1016/j.cnsns.2010.11. 026

25. Li, G., Shi, G., Zhu, Z.H.: Three-dimensional high-fidelity dynamic modeling of tether transportation system with multiple climbers. J. Guid. Control Dyn. 42(8), 1797-1811 (2019). https://doi.org/10.2514/1.g004118
26. Williams, P.: Deployment/retrieval optimization for flexible tethered satellite systems. Nonlinear Dyn. 52(1-2), 159-179 (2008). https://doi.org/10.1007/s11071-007-9269-3

27. Luo, C., Wen, H., Jin, D.: Deployment of flexible space tether system with satellite attitude stabilization. Acta Astronaut. 160(July), 240-250 (2019). https://doi.org/10. 1016/j.actaastro.2019.04.036

28. Li, G., Zhu, Z.H.: Dynamics of partial space elevator with parallel tethers and multiple climbers. Proc. Int. Conf. Aerosp. Syst. Sci. Eng. Lecture Notes Electr. Eng. 622, 231-252 (2019). https://doi.org/10.1007/978-981-15-1773$0 \_18$

29. Shi, G., Li, G., Zhu, Z., Zhu, Z.H.: A virtual experiment for partial space elevator using a novel high-fidelity FE model. Nonlinear Dyn. 95(4), 2717-2727 (2019). https://doi.org/ 10.1007/s11071-018-4718-8

30. Li, G., Zhu, Z.H., Du, C.: Flight dynamics and control strategy of electric solar wind sails. J. Guid. Control Dyn. 43(3), 462-474 (2020). https://doi.org/10.2514/1.g004608

31. Alessandro, A.Q., Giovanni, M., Pekka, J.: Electric sail for a near-Earth asteroid sample return mission: case 1998 KY26. J. Aerosp. Eng. (2014). https://doi.org/10.1061/(ASCE)AS. 1943-5525.0000285

32. Li, G., Zhu, Z.H.: Precise analysis of deorbiting by electrodynamic tethers using coupled multiphysics finite elements. J. Guid. Control Dyn. 40(12), 3343-3352 (2017). https://doi.org/10.2514/1.G002738

33. Li, G., Zhu, Z.H., Meguid, S.A.: Libration and transverse dynamic stability control of flexible bare electrodynamic tether systems in satellite deorbit. Aerosp. Sci. Technol. 49(February), 112-129 (2016). https://doi.org/10.1016/j. ast.2015.11.036

34. Long-Life, S., Jyh-Ching, J., Chen-Tsung, L., Ying-Wen, J.: Spacecraft robust attitude tracking design: PID control approach. In: Proceedings of the 2002 American Control Conference (IEEE Cat. No. CH37301), vol. 2, pp. 1360-1365 (2002). https://doi.org/10.1109/ACC.2002. 1023210

35. Li, G., Zhu, Z.H., Cain, J., Newland, F., Czekanski, A.: Libration control of bare electrodynamic tethers considering elastic-thermal-electrical coupling. J. Guid. Control Dyn. 39(3), 642-654 (2015). https://doi.org/10.2514/1.G001338

36. Guang, Z., Xingzi, B., Bin, L.: Optimal deployment of spinstabilized tethered formations with continuous thrusters. Nonlinear Dyn. 95(3), 2143-2162 (2019). https://doi.org/ $10.1007 / \mathrm{s} 11071-018-4682-3$

Publisher's Note Springer Nature remains neutral with regard to jurisdictional claims in published maps and institutional affiliations. 